\title{
Seasonal Variability in the Essential Oils of Wild and Cultivated Baccharis trimera
}

\author{
Fabiano G. Silva, ${ }^{a}$ Carolina B. A. Oliveira, ${ }^{b}$ José Eduardo B. P. Pinto, ${ }^{c}$ Vivian E. Nascimento, ${ }^{c}$ \\ Suzana C. Santos, ${ }^{b}$ José C. Seraphin ${ }^{d}$ and Pedro H. Ferri ${ }^{*, b}$ \\ ${ }^{a}$ Laboratório de Cultura de Tecidos, Centro Federal de Educação Tecnológica de Rio Verde, CP 66, \\ 75900-000 Rio Verde-GO, Brazil \\ ${ }^{b}$ Laboratório de Bioatividade Molecular, Instituto de Química, Universidade Federal de Goiás, CP 131, \\ 74001-970 Goiânia-GO, Brazil \\ ${ }^{c}$ Laboratório de Cultura de Tecidos e Plantas Medicinais, Departamento de Agricultura, \\ Universidade Federal de Lavras, CP 37, 37200-000 Lavras-MG, Brazil \\ ${ }^{d}$ Núcleo de Estatística Aplicada, Instituto de Matemática e Estatística, Universidade Federal de Goiás, CP 131, \\ 74001-970 Goiânia-GO, Brazil
}

\begin{abstract}
A variação sazonal na composição química do óleo essencial em populações de Baccharis trimera natural e cultivada indicou a presença de três grupos de óleos em relação à origem e à fase de desenvolvimento das amostras. O primeiro incluiu amostras floridas, silvestres ou cultivadas, no período entre março-maio, contendo altas percentagens de globulol e espatulenol. No grupo II, com amostras silvestres coletadas entre junho-fevereiro, os constituintes majoritários foram o germacreno D e o $(E)$-cariofileno, enquanto que o grupo III incluiu amostras cultivadas entre junho-fevereiro e contendo um alto conteúdo de ledol. A análise por correlação canônica indicou que $(E)$-cariofileno, Mn, saturação por Al e precipitação foram fortemente correlacionados às amostras silvestres (grupo II), enquanto ledol e guaiol correlacionaram-se ao balanço químico do solo ( $\mathrm{P}, \mathrm{K}, \mathrm{S}, \mathrm{Cu}, \mathrm{Zn}$ e saturação por bases) nas amostras cultivadas (grupo III). Ambos os óleos essenciais tem predominantemente hidrocarbonetos, embora em dois meses o conteúdo em sesquiterpenos oxigenados seja superior a $40 \%$ para ambas as amostras. As variações observadas podem estar relacionadas ao ambiente.
\end{abstract}

The seasonal variations in the chemical composition of the essential oil of wild and cultivated Baccharis trimera populations indicated the presence of three clusters of oils regarding population and phenophase. The first included flowering, wild, and cultivated samples from a period of March-May, with high percentages of globulol and spathulenol. In cluster II, with wild samples collected from June-February, the major constituents were germacrene D and $(E)$-caryophyllene, while cluster III included cultivated samples from June-February containing a high content of ledol. The canonical correlation analysis revealed that $(E)$-caryophyllene, $\mathrm{Mn}, \mathrm{Al}$ saturation, and precipitation were quite strongly related to wild samples (cluster II), whereas ledol and guaiol were related to chemical balance in soils $(\mathrm{P}, \mathrm{K}, \mathrm{S}, \mathrm{Cu}, \mathrm{Zn}$, and base saturation) of cultivated samples (cluster III). Both essential oils have predominantly hydrocarbon compositions, although for a couple of months the oxygenated sesquiterpene content is over $40 \%$ for both samples. The observed chemovariation might be environmentally determined.

Keywords: Baccharis trimera, Asteraceae, essential oil, chemical variability, seasonal variation

\section{Introduction}

Baccharis species (Asteraceae) are widespread in South America and are known as 'carqueja'. Several

*e-mail: pedro@quimica.ufg.br species are used in traditional medicine or are reputed as toxic to cattle and sheep. ${ }^{1}$ Medicinal teas prepared from the flowering $B$. trimera (Less.) DC. (synon. B. genistelloides var. trimera (Less.) Baker) are used to treat gastrointestinal, renal and liver diseases, diabetes, rheumatism, and may act as anti-inflammatory. ${ }^{2}$ Other 
popular uses in Brazil show that B. trimera is employed in the treatment of malaria, sore throat and tonsillitis, angines, anaemia, urinary inflammation and leprosy. ${ }^{2-4}$ Liver-protective properties, gastrointestinal action, potential antidiabetic activity, and anti-arthritic effects have been validated, 5,6 and the anti-ophidian, relaxant, antimutagenic, antimicrobial, and antiprotozoal activities have also been reported. ${ }^{7,8}$

Chemical studies revealed the presence of clerodanetype diterpenes, saponins and their glycosides, and several flavonoids in this species. ${ }^{5,7}$ Carqueja oil, a commercial fragrance cited as a reminiscent of rosewood, ${ }^{9}$ is stemdistilled from its aerial parts, with the unusual monoterpene alcohol carquejol and its acetyl ester as major components. ${ }^{10-17}$ The commercial value of this oil which is produced in Brazil has been associated with the high content of carquejyl acetate which has showed variations of $30 \%$ to $69.2 \% .^{3,9,14}$ Carquejyl acetate was found in $B$. trimera which was collected in different places of southern Brazil and Argentina, and has been regarded as a chemomarker of this species, ${ }^{17}$ although it has been absent in collected samples in Paraná and Santa Catarina states, South Brazil, ${ }^{18}$ and identified in other species of the same genera. ${ }^{15}$

Despite the great demand of $B$. trimera in the phytotherapeutic industry, ${ }^{18,19}$ as well as the demand for fragrance - B. trimera's essential oil is cited as one of the ten most consumed oils by cosmetic industries in Brazil ${ }^{20}$ the variability in essential oil of wild and cultivated plants has not yet been obtained. ${ }^{21}$ In fact, natural populations have been excessively exploited and their natural habitats have been replaced by commercial crops. As a result, the amount of this wild plant has decreased. ${ }^{21}$

As part of our ongoing work on the characterization of essential oils of medicinal aromatic plants growing wild in the Brazilian Cerrado, ${ }^{22}$ we now report on the results obtained for the essential oil composition and seasonal variability of $B$. trimera that was collected from wild and cultivated populations during a 1-year period. For this purpose, essential oils from aerial parts of representative population samples of each origin were analyzed by GCMS. In order to study chemical variability, chemical constituents were submitted to Principal Component, chemometric Cluster, and Canonical Discriminant analysis in order to detect the pattern distribution of samples and to identify which constituents can distinguish between these groups of individuals. In addition, environmental factors affecting essential oil variability were studied through the application of a Canonical Correlation analysis between the oil component data set and the edaphicclimactic data matrix with 26 variables for each sampling.

\section{Results and Discussion}

B. trimera essential oils were obtained from wild and from cultivated populations. The annual mean yield of oils from wild samples was higher than that of the cultivated plants. Previous work has indicated that organic fertilizer and shade showed no significant effects on the essential oil yield, ${ }^{21,23}$ but radiation increased the content of essential oils in cultivated carqueja. ${ }^{24}$ The seasonal dynamics of essential oil yield in both populations were similar to those of other Cerrado species, which revealed lower values during the dry winter. ${ }^{25}$ In total, 26 compounds were identified, accounting for $96-100 \%$ of the volatile constituents (Table 1). Both essential oils have predominantly sesquiterpene hydrocarbon compositions, although for a couple of months the oxygenated sesquiterpene content is over $40 \%$ for both samples. Essential oils from the Baccharis species have showed that, although the main constituent may vary, sesquiterpenes are usually the dominant class. ${ }^{26}$ Furthermore, important differences in the amounts of the major constituents were found, mainly of germacrene D (6.3-28\%) which had the highest percentage in September-November (both populations); ledol (2.5-16\%) showed a high variation in the wild population, while cultivated samples revealed higher amounts, regardless of the months; and bicyclogermacrene (12-24\%) had the highest contents between July-February in the wild population, and in December-February in cultivated ones.

The results obtained from PCA and nearest neighbour complete linkage cluster analysis using Ward's technique revealed the existence of a high chemical variability within the essential oils of $B$. trimera. ${ }^{27}$ Figure 1 shows the relative position of the individuals in the discriminant space in relation to an axial system that was originated in the PCA. First PC accounts for $c a .47 \%$ of the total variance and separates $(p<0.0001)$ the oxygenated sesquiterpenes of flowering sampling (March-May) from vegetative sampling, regardless of the populations, while the Second PC distinguishes $(p<0.0001)$ the wild sampling from that of the cultivated population in the June-April period. Therefore, three types of essential oils were found: cluster I (wild and cultivated samples harvested in March-May), being characterised by a high percentage $(p<0.0001)$ of globulol $(8.9 \pm 3.4 \%)$, spathulenol $(8.7 \pm 3.1 \%)$, $\alpha$-guaieno (3.4 $\pm 0.4 \%)$, viridiflorol $(3.4 \pm 0.9 \%)$, guaiol $(3.1 \pm 0.8 \%)$, and $\delta$-cadinene $(6.6 \pm 1.9 \%)(p<0.007)$; cluster II (wild samples harvested between June-February) with ( $p<$ $0.0001)$ germacrene $\mathrm{D}(26 \pm 3 \%)$ and $(E)$-caryophyllene 
Table 1. Mean values ${ }^{a}$ of volatile components from populations of cultivated or nature-collected $B$. trimera during the year

\begin{tabular}{|c|c|c|c|c|c|c|c|c|c|c|c|c|c|c|}
\hline Constituents & KI & Population & January & February & March & April & May & June & July & August & September & October & November & December \\
\hline \multirow[t]{2}{*}{$\alpha$-Copaene } & 1376 & Cultivated & $1.3^{\mathrm{Ba}}$ & $1.1^{\mathrm{Ba}}$ & $1.3^{\mathrm{Ba}}$ & $2.4^{\mathrm{Aa}}$ & $2.0^{\mathrm{Aa}}$ & $0.56^{\mathrm{Ca}}$ & $1.6^{\mathrm{Aa}}$ & $0.50^{\mathrm{Ca}}$ & $1.4^{\mathrm{Ba}}$ & $2.0^{\mathrm{Aa}}$ & $1.6^{\mathrm{Aa}}$ & $0.99 \mathrm{Ba}$ \\
\hline & & Wild & $1.7^{\mathrm{Aa}}$ & $1.3^{\mathrm{Aa}}$ & $1.2^{\mathrm{Aa}}$ & $1.4^{\mathrm{Ab}}$ & $1.7^{\mathrm{Aa}}$ & $1.2^{\mathrm{Aa}}$ & $0.97 \mathrm{Aa}$ & $0.93 \mathrm{Aa}$ & $1.3^{\mathrm{Aa}}$ & $1.5^{\mathrm{Aa}}$ & $1.7^{\mathrm{Aa}}$ & $1.6^{\mathrm{Aa}}$ \\
\hline \multirow[t]{2}{*}{$\beta$-Cubebene } & 1390 & Cultivated & - & - & - & $0.22^{\mathrm{A}}$ & - & - & $0.13^{\mathrm{Aa}}$ & - & - & $0.24^{\mathrm{Aa}}$ & - & - \\
\hline & & Wild & $0.06^{\text {в }}$ & - & - & - & - & - & 0.26 Аa & $0.01^{\text {в }}$ & $0.13^{\mathrm{A}}$ & $0.25^{\mathrm{Aa}}$ & $0.14^{\mathrm{A}}$ & - \\
\hline \multirow[t]{2}{*}{$\beta$-Elemene } & 1392 & Cultivated & $0.77^{\mathrm{Ca}}$ & $0.36^{\mathrm{Ca}}$ & $0.01^{\mathrm{Ca}}$ & $0.99^{\mathrm{Ca}}$ & $0.14^{\mathrm{Ca}}$ & $0.86^{\mathrm{Ba}}$ & $0.62 \mathrm{Ba}$ & $0.60^{\mathrm{Ba}}$ & $0.81^{\mathrm{Ba}}$ & $1.2^{\mathrm{Aa}}$ & $0.37^{\mathrm{Cb}}$ & $0.57 \mathrm{Ba}$ \\
\hline & & Wild & $0.21^{\mathrm{Ca}}$ & $0.54^{\mathrm{Ba}}$ & $0.01^{\mathrm{Ca}}$ & $0.01^{\mathrm{Ca}}$ & $0.44^{\mathrm{Ba}}$ & $0.62^{\mathrm{Ba}}$ & $0.74^{\mathrm{Aa}}$ & $0.59^{\text {ва }}$ & $0.63^{\mathrm{Ba}}$ & $0.81^{\mathrm{Ab}}$ & $1.1^{\mathrm{Aa}}$ & $0.81^{\mathrm{Aa}}$ \\
\hline \multirow[t]{2}{*}{$\alpha$-Gurjunene } & 1410 & Cultivated & $0.59^{\mathrm{Aa}}$ & $0.94 \mathrm{Aa}$ & $0.24 \mathrm{Ba}$ & $0.25^{\mathrm{Ba}}$ & $0.31^{\mathrm{Ba}}$ & $0.90 \mathrm{Aa}$ & 0.69 Аa & $0.63 \mathrm{Aa}$ & $0.51^{\mathrm{Aa}}$ & $0.59^{\mathrm{Aa}}$ & $0.54 \mathrm{Aa}$ & $0.66^{\mathrm{Aa}}$ \\
\hline & & Wild & $0.24^{\mathrm{Ab}}$ & $0.18^{\mathrm{Ab}}$ & $0.15^{\mathrm{Aa}}$ & $0.11^{\mathrm{Aa}}$ & $0.23^{\mathrm{Aa}}$ & $0.47^{\mathrm{Ab}}$ & $0.20^{\mathrm{Ab}}$ & $0.31 \mathrm{Aa}$ & $0.37^{\mathrm{Ab}}$ & $0.11^{\mathrm{Ab}}$ & - & $0.25^{\mathrm{Ab}}$ \\
\hline \multirow{2}{*}{$\begin{array}{l}(E) \text {-Caryo- } \\
\text { phyllene }\end{array}$} & 1420 & Cultivated & $16^{\mathrm{Ab}}$ & $17^{\mathrm{Ab}}$ & $16^{\mathrm{Aa}}$ & $16^{\mathrm{Aa}}$ & $17 \mathrm{Aa}$ & $14^{\text {вь }}$ & $13^{\mathrm{Bb}}$ & $12^{\mathrm{Bb}}$ & $14^{\mathrm{Ba}}$ & $14^{\mathrm{Ba}}$ & $14^{\mathrm{Ba}}$ & $16^{\mathrm{Ab}}$ \\
\hline & & Wild & $21^{\mathrm{Aa}}$ & 20 Aa & $16^{\mathrm{Ba}}$ & $16^{\mathrm{Ba}}$ & $19^{\mathrm{Aa}}$ & $19^{\mathrm{Aa}}$ & $17^{\text {Ва }}$ & $17^{\mathrm{Ba}}$ & $17^{\text {Ва }}$ & $16^{\mathrm{Ba}}$ & $17^{\mathrm{Ba}}$ & $20^{\mathrm{Aa}}$ \\
\hline \multirow[t]{2}{*}{$\alpha$-Guaiene } & 1439 & Cultivated & $2.4^{\mathrm{Ba}}$ & $2.4^{\mathrm{Ba}}$ & $3.4^{\mathrm{Aa}}$ & $3.7^{\mathrm{Aa}}$ & $3.7^{\mathrm{Aa}}$ & $2.4^{\mathrm{Ba}}$ & $1.9^{\mathrm{Ca}}$ & $1.9^{\mathrm{Ca}}$ & $1.7^{\mathrm{Ca}}$ & $1.6^{\mathrm{Ca}}$ & $2.2^{\mathrm{Ba}}$ & $2.5^{\mathrm{Ba}}$ \\
\hline & & Wild & $2.6^{\mathrm{Ca}}$ & $3.0^{\mathrm{Ba}}$ & $3.3^{\mathrm{Aa}}$ & $3.8^{\mathrm{Aa}}$ & $2.8^{\mathrm{Bb}}$ & $2.5^{\mathrm{Ca}}$ & $1.9^{\mathrm{Ba}}$ & $2.0^{\mathrm{Ba}}$ & $1.9^{\mathrm{Da}}$ & $2.1^{\mathrm{Da}}$ & $2.4^{\mathrm{Ca}}$ & $2.9^{\mathrm{Ba}}$ \\
\hline \multirow[t]{2}{*}{$\alpha$-Humulene } & 1454 & Cultivated & $1.4^{\mathrm{Bb}}$ & $1.4^{\mathrm{Bb}}$ & $1.8^{\mathrm{Aa}}$ & $1.9^{\mathrm{Aa}}$ & $1.6^{\mathrm{Ab}}$ & $1.3^{\mathrm{Bb}}$ & $1.4^{\mathrm{Ba}}$ & $1.2^{\mathrm{Bb}}$ & $1.4^{\mathrm{Ba}}$ & $1.6^{\mathrm{Bb}}$ & $1.2^{\mathrm{Bb}}$ & $1.4^{\mathrm{Bb}}$ \\
\hline & & Wild & $1.9^{\mathrm{Aa}}$ & $1.8^{\mathrm{Ba}}$ & $2.0^{\mathrm{Aa}}$ & $1.8^{\mathrm{Ba}}$ & $1.9^{\mathrm{Aa}}$ & $1.7^{\mathrm{Ba}}$ & $1.6^{\mathrm{Ba}}$ & $1.6^{\mathrm{Ba}}$ & $1.6^{\mathrm{Ba}}$ & $1.9^{\mathrm{Aa}}$ & $1.8^{\mathrm{Ba}}$ & $2.1^{\mathrm{Aa}}$ \\
\hline \multirow[t]{2}{*}{$\gamma$-Gurjunene } & 1472 & Cultivated & $0.55^{\mathrm{Aa}}$ & $0.60^{\mathrm{Aa}}$ & $0.27^{\mathrm{Aa}}$ & $0.11^{\mathrm{A}}$ & 0.94 Aa & $0.86^{\mathrm{Aa}}$ & $0.69^{\mathrm{A}}$ & $0.58^{\mathrm{Aa}}$ & $0.37^{\mathrm{A}}$ & $0.54^{\mathrm{A}}$ & $1.1^{\mathrm{Aa}}$ & $0.73^{\mathrm{Aa}}$ \\
\hline & & Wild & $0.08 \mathrm{Aa}$ & $0.07^{\mathrm{Aa}}$ & $0.10^{\mathrm{Aa}}$ & - & $0.92 \mathrm{Aa}$ & $0.30^{\mathrm{Aa}}$ & - & $0.16^{\mathrm{Aa}}$ & - & - & $0.46^{\mathrm{Aa}}$ & $0.27^{\mathrm{Aa}}$ \\
\hline \multirow[t]{2}{*}{$\gamma$-Muurolene } & 1477 & Cultivated & $1.6^{\mathrm{Ca}}$ & $1.4^{\mathrm{Ca}}$ & $2.1^{\mathrm{Ca}}$ & $3.8^{\mathrm{Aa}}$ & $2.5^{\mathrm{Ba}}$ & $1.1^{\mathrm{Ca}}$ & $1.2^{\mathrm{Ca}}$ & $0.9^{\mathrm{Ca}}$ & $1.3^{\mathrm{Ca}}$ & $1.2^{\mathrm{Ca}}$ & $1.6^{\mathrm{Ca}}$ & $1.4^{\mathrm{Cb}}$ \\
\hline & & Wild & $2.2^{\mathrm{Ba}}$ & $2.0^{\mathrm{Ba}}$ & $2.0^{\mathrm{Ba}}$ & $3.0^{\mathrm{Ab}}$ & $2.6^{\mathrm{Aa}}$ & $1.4^{\mathrm{Ca}}$ & $0.97^{\mathrm{Ca}}$ & $1.0^{\mathrm{Ca}}$ & $1.2^{\mathrm{Ca}}$ & $1.2^{\mathrm{Ca}}$ & $1.4^{\mathrm{Ca}}$ & $2.1^{\mathrm{Ba}}$ \\
\hline \multirow[t]{2}{*}{ Germacrene D } & 1483 & Cultivated & $25^{\mathrm{Aa}}$ & $25^{\mathrm{Aa}}$ & $7.7^{\mathrm{Da}}$ & $12^{\mathrm{Ca}}$ & $6.3^{\mathrm{Db}}$ & $18^{\mathrm{Ba}}$ & $22^{\mathrm{Ab}}$ & $20^{\mathrm{Bb}}$ & $25^{\mathrm{Aa}}$ & $26^{\mathrm{Aa}}$ & $23^{\text {Aa }}$ & $20^{\mathrm{Ba}}$ \\
\hline & & Wild & $24 \mathrm{Ba}$ & $27^{\mathrm{Aa}}$ & $8.9^{\mathrm{Ca}}$ & $12^{\mathrm{Ca}}$ & $13^{\mathrm{Ca}}$ & $20^{\mathrm{Ba}}$ & $27^{\mathrm{Aa}}$ & $27^{\mathrm{Aa}}$ & $28 \mathrm{Aa}$ & $28^{\mathrm{Aa}}$ & $26^{\mathrm{Aa}}$ & $23^{\mathrm{Ba}}$ \\
\hline \multirow[t]{2}{*}{$\beta$-Selinene } & 1487 & Cultivated & $0.75^{\mathrm{Ba}}$ & $0.74^{\mathrm{Ba}}$ & $1.2^{\mathrm{Aa}}$ & $1.3^{\mathrm{Aa}}$ & $1.1^{\mathrm{Aa}}$ & $0.62 \mathrm{Ba}$ & - & 0.52 ва & $0.57 \mathrm{Ba}$ & $0.41 \mathrm{Ba}$ & 0.90 Aa & $0.93 \mathrm{Aa}$ \\
\hline & & Wild & 0.79 ва & $0.77^{\mathrm{Ba}}$ & $1.3^{\mathrm{Aa}}$ & $1.1^{\mathrm{Aa}}$ & $0.86^{\mathrm{Ba}}$ & $0.85^{\mathrm{Ba}}$ & $0.41^{\mathrm{C}}$ & $0.49^{\mathrm{Ca}}$ & $0.60^{\mathrm{Ca}}$ & $0.67^{\mathrm{Ca}}$ & $0.76^{\mathrm{Ba}}$ & $0.91 \mathrm{Ba}$ \\
\hline \multirow{2}{*}{$\begin{array}{l}\text { Bicyclo- } \\
\text { germacrene }\end{array}$} & 1497 & Cultivated & $23^{\mathrm{Aa}}$ & $23^{\mathrm{Aa}}$ & $14^{\mathrm{Ba}}$ & $14^{\mathrm{Ba}}$ & $12^{\text {Ва }}$ & $20^{\mathrm{Aa}}$ & $17^{\mathrm{Bb}}$ & $24^{\mathrm{Aa}}$ & $16^{\mathrm{Bb}}$ & $15^{\mathrm{Bb}}$ & $15^{\mathrm{Bb}}$ & $21^{\mathrm{Aa}}$ \\
\hline & & Wild & $20^{\mathrm{Aa}}$ & $22^{\mathrm{Aa}}$ & $15^{\mathrm{Ba}}$ & $15^{\mathrm{Ba}}$ & $13^{\mathrm{Ba}}$ & $17^{\mathrm{Ba}}$ & $23^{\mathrm{Aa}}$ & $23^{\mathrm{Aa}}$ & $21^{\mathrm{Aa}}$ & $22^{\mathrm{Aa}}$ & $20^{\mathrm{Aa}}$ & $19^{\mathrm{Aa}}$ \\
\hline$\alpha$-Muurulene & 1500 & Cultivated & $1.0^{\mathrm{Ba}}$ & $0.88^{\mathrm{Ba}}$ & $1.1^{\mathrm{Ba}}$ & $2.1^{\mathrm{Aa}}$ & $1.7^{\mathrm{Aa}}$ & $0.48^{\mathrm{Bb}}$ & $0.80^{\mathrm{Ba}}$ & $0.45^{\mathrm{Ba}}$ & $0.78^{\mathrm{Ba}}$ & $0.86^{\mathrm{Ba}}$ & $0.82^{\text {ва }}$ & $0.90^{\mathrm{Ba}}$ \\
\hline & & Wild & $1.3^{\mathrm{Aa}}$ & $1.1^{\mathrm{Ba}}$ & $1.0^{\mathrm{Ba}}$ & $1.5^{\mathrm{Ab}}$ & $1.5^{\mathrm{Aa}}$ & $0.92^{\mathrm{Ba}}$ & $0.52^{\mathrm{Ba}}$ & $0.68^{\text {ва }}$ & $0.88^{\mathrm{Ba}}$ & $0.71^{\mathrm{Ba}}$ & $1.1 \mathrm{Ba}$ & $1.1^{\mathrm{Ba}}$ \\
\hline$\alpha$-Bulnecene & 1506 & Cultivated & $1.0^{\mathrm{Aa}}$ & $1.0^{\mathrm{Aa}}$ & $0.43 \mathrm{Ba}$ & $0.34^{\mathrm{Ba}}$ & $0.10^{\mathrm{Bb}}$ & $1.2^{\mathrm{Aa}}$ & $0.92 \mathrm{Aa}$ & $1.2^{\mathrm{Aa}}$ & $0.99 \mathrm{Aa}$ & $1.1^{\mathrm{Aa}}$ & $0.73^{\mathrm{Ab}}$ & $0.86^{\mathrm{Aa}}$ \\
\hline & & Wild & $1.1^{\mathrm{Aa}}$ & $1.1^{\mathrm{Aa}}$ & $0.39 \mathrm{Ba}$ & $0.46^{\mathrm{Ba}}$ & $0.45^{\mathrm{Ba}}$ & $1.2^{\mathrm{Aa}}$ & 0.95 Аa & $1.3^{\mathrm{Aa}}$ & $1.2^{\mathrm{Aa}}$ & $1.3^{\mathrm{Aa}}$ & $1.1^{\mathrm{Aa}}$ & $0.92 \mathrm{Aa}$ \\
\hline$\gamma$-Cadinene & 1515 & Cultivated & $0.48 \mathrm{Aa}$ & $0.28^{\mathrm{Ba}}$ & $0.76^{\mathrm{Aa}}$ & $1.3^{\mathrm{Aa}}$ & $1.0^{\mathrm{Aa}}$ & - & $0.72^{\mathrm{A}}$ & - & - & $0.08^{\mathrm{Ba}}$ & $0.31 \mathrm{Ba}$ & $0.43^{\mathrm{Ba}}$ \\
\hline & & Wild & $0.85^{\mathrm{Aa}}$ & $0.77^{\mathrm{Aa}}$ & $0.77^{\mathrm{Aa}}$ & $1.3^{\mathrm{Aa}}$ & 0.87 Аa & $0.14^{\text {в }}$ & - & $0.05^{\text {в }}$ & $0.25^{\text {в }}$ & $0.07^{\mathrm{Ba}}$ & $0.54^{\mathrm{Aa}}$ & $0.644^{\mathrm{Aa}}$ \\
\hline$\delta$-Cadinene & 1523 & Cultivated & $5.5^{\mathrm{Aa}}$ & $4.2^{\mathrm{Ba}}$ & $5.8^{\mathrm{Aa}}$ & $8.7^{\mathrm{Aa}}$ & $5.9^{\mathrm{Aa}}$ & $3.8^{\mathrm{Ba}}$ & $5.1^{\mathrm{Ba}}$ & $3.0^{\mathrm{Ba}}$ & $6.4^{\mathrm{Aa}}$ & $6.1^{\mathrm{Aa}}$ & $6.2^{\mathrm{Aa}}$ & $4.7^{\mathrm{Bb}}$ \\
\hline & & Wild & $6.2^{\mathrm{Aa}}$ & $6.3^{\mathrm{Aa}}$ & $6.1^{\mathrm{Aa}}$ & $5.2^{\mathrm{Ab}}$ & $6.8^{\mathrm{Aa}}$ & $5.1^{\mathrm{Aa}}$ & $5.0^{\mathrm{Aa}}$ & $4.5^{\mathrm{Aa}}$ & $6.0^{\mathrm{Aa}}$ & $6.4^{\mathrm{Aa}}$ & $7.4^{\mathrm{Aa}}$ & $7.3^{\mathrm{Aa}}$ \\
\hline$\delta$-Calacorene & 1544 & Cultivated & - & - & - & - & - & - & - & - & - & - & - & - \\
\hline & & Wild & - & - & 0.16 & - & - & - & - & - & - & - & - & - \\
\hline Germacrene B & 1558 & Cultivated & $0.23^{\mathrm{Ba}}$ & $0.18^{\mathrm{Ba}}$ & - & - & $0.15^{\mathrm{Ba}}$ & $0.06^{\mathrm{Ba}}$ & $2.2^{\mathrm{Aa}}$ & $0.28^{\mathrm{Ba}}$ & $0.06^{\mathrm{Ba}}$ & - & $0.18^{\mathrm{Ba}}$ & $0.05^{\mathrm{Ba}}$ \\
\hline & & Wild & $0.30^{\mathrm{Aa}}$ & $0.36^{\mathrm{Aa}}$ & - & $1.2^{\mathrm{A}}$ & $0.31^{\mathrm{Aa}}$ & $0.27^{\mathrm{Aa}}$ & $0.16^{\mathrm{Ab}}$ & $0.64{ }^{\mathrm{Aa}}$ & $0.36^{\mathrm{Aa}}$ & $0.28^{\mathrm{A}}$ & $0.53^{\mathrm{Aa}}$ & $0.52^{\mathrm{Aa}}$ \\
\hline Ledol & 1570 & Cultivated & $7.5^{\mathrm{Ba}}$ & $10^{\mathrm{Aa}}$ & 12 Aa & $4.2^{\mathrm{Ba}}$ & $9.7^{\mathrm{Aa}}$ & $16^{\mathrm{Aa}}$ & $12 \mathrm{Aa}$ & $13^{\mathrm{Aa}}$ & $11^{\mathrm{Aa}}$ & $12^{\mathrm{Aa}}$ & $9.6^{\text {Aa }}$ & $12^{\mathrm{Aa}}$ \\
\hline & & Wild & $2.9^{\mathrm{Ba}}$ & $2.8^{\mathrm{Ba}}$ & $9.6^{\mathrm{Aa}}$ & $3.6^{\mathrm{Ba}}$ & $9.3^{\mathrm{Aa}}$ & $8.6^{\mathrm{Ab}}$ & $5.9^{\mathrm{Bb}}$ & $7.2^{\mathrm{Ab}}$ & $4.8^{\mathrm{Bb}}$ & $6.1^{\mathrm{Bb}}$ & $4.4^{\mathrm{Bb}}$ & $4.1^{\mathrm{Bb}}$ \\
\hline Spathulenol & 1578 & Cultivated & $2.6^{\mathrm{Da}}$ & $1.8^{\mathrm{Da}}$ & $12^{\mathrm{Aa}}$ & $5.2^{\mathrm{Ca}}$ & $9.4^{\mathrm{Ba}}$ & $6.6^{\mathrm{Ca}}$ & $6.4^{\mathrm{Ca}}$ & $6.3^{\mathrm{Ca}}$ & $5.1^{\mathrm{Ca}}$ & $3.1^{\mathrm{Da}}$ & $3.0^{\mathrm{Da}}$ & $4.0^{\mathrm{Da}}$ \\
\hline & & Wild & $3.0^{\mathrm{Ca}}$ & $1.2^{\mathrm{Ca}}$ & $12^{\mathrm{Aa}}$ & $6.6^{\mathrm{Ba}}$ & $7.1^{\mathrm{Bb}}$ & $6.4^{\mathrm{Ba}}$ & $3.5^{\mathrm{Cb}}$ & $3.7^{\mathrm{Cb}}$ & $3.3^{\mathrm{Ca}}$ & $2.1^{\mathrm{Ca}}$ & $2.5^{\mathrm{Ca}}$ & $3.1^{\mathrm{Ca}}$ \\
\hline Globulol & 1583 & Cultivated & $3.8^{\mathrm{Da}}$ & $3.1^{\mathrm{Da}}$ & $8.3^{\mathrm{Ba}}$ & $8.6^{\mathrm{Ba}}$ & $11^{\mathrm{Aa}}$ & $4.8^{\mathrm{Ca}}$ & $5.8^{\mathrm{Ca}}$ & $6.2^{\mathrm{Ca}}$ & $6.2^{\mathrm{Ca}}$ & $5.0^{\mathrm{Ca}}$ & $3.0^{\mathrm{Da}}$ & $4.7^{\mathrm{Ca}}$ \\
\hline & & Wild & $4.2^{\mathrm{Ba}}$ & $2.9^{\mathrm{Ba}}$ & $8.8^{\mathrm{Aa}}$ & $8.9^{\mathrm{Aa}}$ & $6.9^{\mathrm{Ab}}$ & $5.1^{\mathrm{Ba}}$ & $4.0^{\mathrm{Ba}}$ & $3.8^{\mathrm{Ba}}$ & $4.3^{\mathrm{Ba}}$ & $3.7^{\mathrm{Ba}}$ & $3.6^{\mathrm{Ba}}$ & $3.9^{\mathrm{Ba}}$ \\
\hline Viridiflorol & 1592 & Cultivated & $1.4^{\mathrm{Ba}}$ & $1.3^{\mathrm{Ba}}$ & $3.5^{\mathrm{Aa}}$ & $3.5^{\mathrm{Aa}}$ & $3.6^{\mathrm{Aa}}$ & $1.5^{\mathrm{Ba}}$ & $1.5^{\mathrm{Ba}}$ & $1.5^{\mathrm{Ba}}$ & $0.74^{\mathrm{Ca}}$ & $0.54^{\mathrm{Ca}}$ & $0.67 \mathrm{Ca}$ & $1.7^{\mathrm{Ba}}$ \\
\hline & & Wild & $2.0^{\mathrm{Ca}}$ & $1.6^{\mathrm{Ca}}$ & $2.9^{\mathrm{Ba}}$ & $4.3^{\mathrm{Aa}}$ & $2.6^{\mathrm{Bb}}$ & $1.4^{\mathrm{Ca}}$ & $1.3^{\mathrm{Ca}}$ & $1.1^{\mathrm{Ca}}$ & $1.5^{\mathrm{Ca}}$ & $1.2^{\mathrm{Ca}}$ & $1.4^{\mathrm{Ca}}$ & $1.7^{\mathrm{Ca}}$ \\
\hline Guaiol & 1604 & Cultivated & $2.2^{\mathrm{Ba}}$ & $2.5^{\mathrm{Ba}}$ & $3.5^{\mathrm{Aa}}$ & $3.5^{\mathrm{Ba}}$ & $3.8^{\mathrm{Aa}}$ & $3.5^{\mathrm{Aa}}$ & $1.9^{\mathrm{Ba}}$ & $2.8^{\mathrm{Ba}}$ & $2.4^{\mathrm{Ba}}$ & $2.6^{\mathrm{Ba}}$ & $2.7^{\mathrm{Ba}}$ & $3.0^{\mathrm{Ba}}$ \\
\hline & & Wild & $0.90^{\mathrm{Bb}}$ & $0.88^{\mathrm{Bb}}$ & $3.3^{\mathrm{Aa}}$ & $3.0^{\mathrm{Aa}}$ & $2.7^{\mathrm{Aa}}$ & $1.7^{\mathrm{Bb}}$ & $1.4^{\mathrm{Ba}}$ & $1.5^{\mathrm{Bb}}$ & $1.2^{\mathrm{Bb}}$ & $0.77^{\mathrm{Bb}}$ & $1.5^{\mathrm{Ba}}$ & $1.0^{\mathrm{Bb}}$ \\
\hline epi- $\alpha$-Cadinol & 1640 & Cultivated & - & - & $1.0^{\mathrm{Aa}}$ & $0.30^{\mathrm{Ab}}$ & $0.48 \mathrm{Aa}$ & $0.45^{\mathrm{Aa}}$ & 0.44 Aa & $0.29 \mathrm{Aa}$ & $0.16^{\mathrm{Aa}}$ & $0.06^{\mathrm{A}}$ & - & $0.42^{\mathrm{Aa}}$ \\
\hline & & Wild & - & $0.41^{\text {в }}$ & 0.94 Aa & $1.5^{\mathrm{Aa}}$ & $0.08^{\text {ва }}$ & $0.33^{\mathrm{Ba}}$ & $0.34^{\mathrm{Ba}}$ & $0.18^{\mathrm{Ba}}$ & $0.06^{\mathrm{Ba}}$ & - & $0.27^{\text {в }}$ & $0.26^{\mathrm{Ba}}$ \\
\hline$\alpha$-Cadinol & 1655 & Cultivated & $1.1^{\mathrm{Ba}}$ & $1.0^{\mathrm{Ba}}$ & $2.4^{\mathrm{Aa}}$ & $2.9^{\mathrm{Ab}}$ & $2.7^{\mathrm{Aa}}$ & $0.99 \mathrm{Ba}$ & $0.76^{\mathrm{Ba}}$ & $0.84^{\mathrm{Ba}}$ & $1.2^{\mathrm{Ba}}$ & $0.95^{\mathrm{Ba}}$ & $0.99 \mathrm{Ba}$ & $1.2^{\mathrm{Ba}}$ \\
\hline & & Wild & $1.6^{\mathrm{Ba}}$ & $1.4^{\mathrm{Ba}}$ & $2.5^{\mathrm{Ba}}$ & $4.7^{\text {Аа }}$ & $2.3^{\mathrm{Ba}}$ & $1.1^{\mathrm{Ba}}$ & $1.3^{\mathrm{Ba}}$ & $0.90^{\mathrm{Ba}}$ & $1.0^{\mathrm{Ba}}$ & $1.5^{\mathrm{Ba}}$ & $1.7^{\mathrm{Ba}}$ & $1.4^{\mathrm{Ba}}$ \\
\hline Eudesma-4(15), & -1691 & Cultivated & $0.37^{\mathrm{Ba}}$ & $0.32 \mathrm{Ba}$ & $0.23^{\mathrm{Ba}}$ & $0.29 \mathrm{Ba}$ & - & - & $0.08^{\text {в }}$ & $0.12^{\mathrm{Ba}}$ & $0.63^{\mathrm{Aa}}$ & $0.81^{\mathrm{Aa}}$ & $0.66^{\mathrm{Aa}}$ & $0.07 \mathrm{Ba}$ \\
\hline dien-1- $\beta$-ol & & Wild & $0.15^{\mathrm{Ba}}$ & $0.12^{\mathrm{Ba}}$ & - & $0.31^{\text {Aa }}$ & $0.08^{\mathrm{B}}$ & - & - & $0.07^{\mathrm{Ba}}$ & $0.54^{\mathrm{Aa}}$ & 0.52 Аа & $0.52^{\mathrm{Aa}}$ & $0.06^{\mathrm{Aa}}$ \\
\hline Sesquiterpene & ydrocarbons & Cultivated & $81^{\text {Aa }}$ & $79^{\mathrm{Ab}}$ & $56^{\mathrm{Ca}}$ & $68^{\mathrm{Ba}}$ & $56^{\mathrm{Cb}}$ & $65^{\mathrm{Ba}}$ & $68^{\mathrm{Bb}}$ & $68^{\mathrm{Bb}}$ & $72^{\mathrm{Bb}}$ & $72^{\mathrm{Bb}}$ & $77^{\text {Aa }}$ & $73^{\mathrm{Bb}}$ \\
\hline & & Wild & $84^{\mathrm{Aa}}$ & $88^{\mathrm{Aa}}$ & $58^{\mathrm{Ca}}$ & $69^{\mathrm{Ba}}$ & $66^{\mathrm{Ba}}$ & $72 \mathrm{Ba}$ & $81^{\mathrm{Aa}}$ & $80^{\mathrm{Aa}}$ & $83^{\mathrm{Aa}}$ & $84^{\mathrm{Aa}}$ & $83^{\mathrm{Aa}}$ & $84^{\mathrm{Aa}}$ \\
\hline Oxygenated se & quiterpenes & Cultivated & $19^{\mathrm{Da}}$ & $20^{\mathrm{Da}}$ & $42 \mathrm{Aa}$ & $27^{\mathrm{Ca}}$ & 41 Aa & $34^{\mathrm{Ba}}$ & $31^{\mathrm{Ca}}$ & $31^{\mathrm{Ca}}$ & $28^{\mathrm{Ca}}$ & $25^{\mathrm{Ca}}$ & $25^{\mathrm{Ca}}$ & $27^{\mathrm{Ca}}$ \\
\hline & & Wild & $15^{\mathrm{Ca}}$ & $11^{\mathrm{Cb}}$ & $40^{\mathrm{Aa}}$ & $28^{\mathrm{Ba}}$ & $32^{\mathrm{Bb}}$ & $25^{\mathrm{Bb}}$ & $18^{\mathrm{cb}}$ & $19^{\mathrm{Cb}}$ & $17^{\mathrm{Cb}}$ & $16^{\mathrm{cb}}$ & $16^{\mathrm{Cb}}$ & $16^{\mathrm{cb}}$ \\
\hline Oil yield/(\%) & & Cultivated & $0.25^{\mathrm{Aa}}$ & $0.27^{\mathrm{Ab}}$ & $0.22 \mathrm{Ba}$ & $0.23 \mathrm{Ba}$ & $0.19^{\mathrm{Ca}}$ & $0.17^{\mathrm{Ca}}$ & $0.13^{\mathrm{Db}}$ & $0.12^{\mathrm{Da}}$ & $0.12^{\mathrm{Da}}$ & $0.11^{\mathrm{Da}}$ & $0.16^{\mathrm{Ca}}$ & $0.15^{\mathrm{Cb}}$ \\
\hline & & Wild & $0.28 \mathrm{Ba}$ & 0.32 Aa & $0.23^{\mathrm{Ca}}$ & $0.23^{\mathrm{Ca}}$ & $0.17^{\mathrm{Da}}$ & $0.16^{\mathrm{Da}}$ & $0.17^{\mathrm{Da}}$ & $0.14^{\mathrm{Ea}}$ & $0.13 \mathrm{Ea}$ & $0.12 \mathrm{Ea}$ & $0.18^{\mathrm{Da}}$ & $0.22^{\mathrm{Ca}}$ \\
\hline
\end{tabular}

${ }^{\text {a }}$ Percentage data. Means followed by the same capital letter in the rows and same small letter in the columns did not share significant differences at $5 \%$ probability by Scott-Knott test. (-): not detected. 
$(19 \pm 2 \%)$ as principal constituents; and cluster III (cultivated samples harvested in the June-February period) containing a high amount of ledol $(12 \pm 3 \%)(p<0.0001)$.

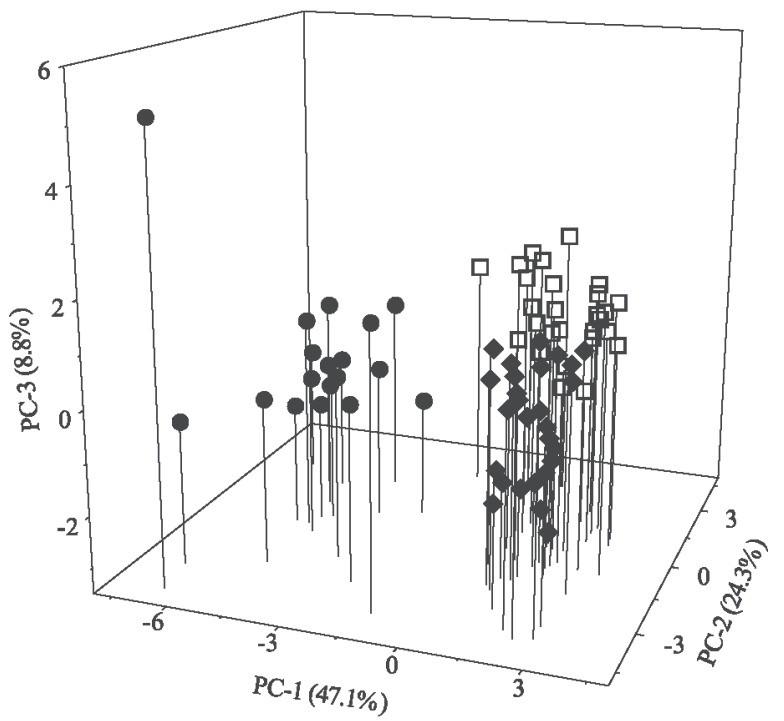

Figure 1. Principal Component scatterplot of B. trimera samples collected from wild and cultivated populations to which cluster it belongs: I $(\bullet)$, II $(\square)$ and III $(\diamond)$.

The canonical discriminant analysis (CDA) confirmed this clustering as a priori groupings and a twodimensional axial system that originated in the CDA distinguished the different types of essential oils based on the contents of globulol, germacrene D and ledol as predictor variables (Figure 2). The first discriminant function (F1) accounts for $c a$. $90 \%$ of the total variability and separates the samples in flowering stage $(p<$ 0.0001 ), regardless of the population, due to the high content of globulol. On the other hand, the second discriminant function (F2) distinguishes the harvested samples according to the wild or cultivated population $(p<0.0003)$, as a result of the high contents of germacrene $\mathrm{D}$ and ledol, respectively. In addition, when using the two discriminant functions it is possible to predict accurately $c a$. $96 \%$ well classification in the original clusters (only one misclassification) by a crossvalidation approach. This approach involves taking a slightly reduced number of samples from the parent data set, estimating parameters from each of these modified data sets and then calculating the precision of the predictions for the samples previously removed by the resulting models. ${ }^{28}$ The only observed misclassification was the cultivated sample harvested in January (Figure 2 ), which was classified as belonging to the wild population. Such misclassification could be caused by lower levels of ledol during January (a characteristic of wild samples).

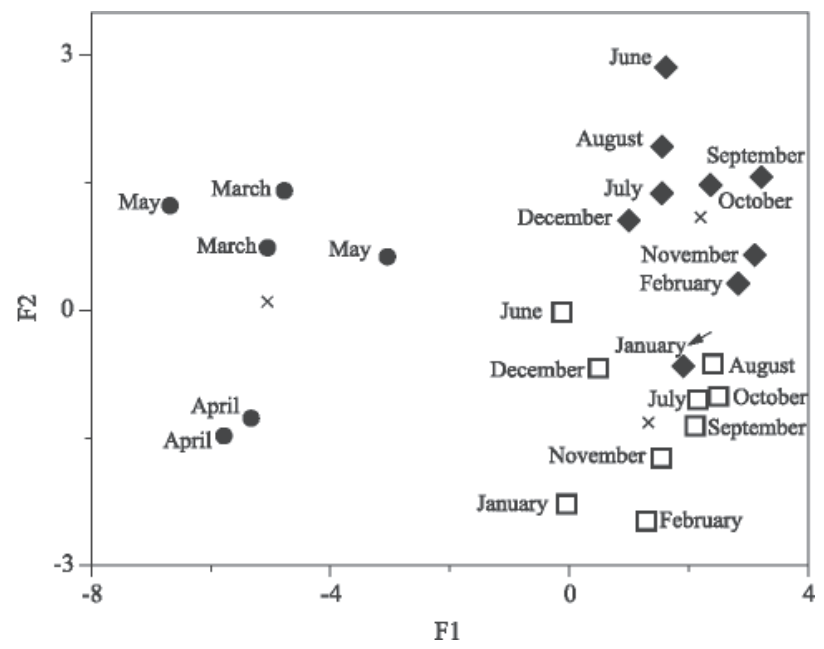

Figure 2. Canonical discriminant-scatterplot of B. trimera sampling months from wild and cultivated populations to which cluster it belongs: I (•), II ( $\square)$ and III ( ). Arrow shows the misclassification individual. Crosses represent the group centroids on the canonical variates.

All these findings may be correlated with factors other than genetic determination as biotic pressures which could modulate the volatiles of cluster I (flowering phase) from clusters II and III (vegetative phase), the former being influenced by pollinators and the latter by pathogens and herbivores or because of differences in environmental conditions. ${ }^{29}$ In fact, the canonical correlation analysis (Table 2) between populations and soils revealed that ledol and guaiol present a strong relationship with chemical balance in soils (P, K, S, Cu, Zn, and base saturation) and are related to the cultivated samples collected in the JuneFebruary period (cluster III). In addition, $(E)$ caryophyllene from the first set and $\mathrm{Mn}, \mathrm{Al}$ saturation, soil humidity, and precipitation from the second set load fairly strongly onto the first canonical variable, which is related to the wild samples collected in the June-February period (cluster II).

Unlike previous studies which used plants that were collected in different places of southern Brazil and Argentina, ${ }^{10-17}$ where carquejol reached $0-9 \%$ and carquejyl acetate $12.2-69.2 \%$, our results did not reveal any of these chemicals. By contrast, germacrene D $(21 \pm$ $7 \%)$, bicyclogermacrene $(19 \pm 4 \%),(E)$-caryophyllene (17 $\pm 3 \%)$, ledol $(8.3 \pm 4.0 \%), \delta$-cadinene $(5.8 \pm 1.6 \%)$, globulol $(5.4 \pm 2.5 \%)$, and spathulenol $(5.0 \pm 3.1 \%)$ were always present as major compounds in our samples, whereas in previous studies some of these chemicals were obtained as minor constituents or were only reported in trace amounts. ${ }^{11-13,16,17}$ Some studies report high contents of ledol $(9.93 \pm 7.34 \%),{ }^{16,17}$ whereas other researches did not find these volatiles at all. ${ }^{10,14,15}$ The lack of carquejol and carquejyl acetate was also reported in samples 
Table 2. Canonical correlation structure (loadings) of the oil components and edapho-climactic factors with their canonical variates

\begin{tabular}{|c|c|c|c|}
\hline $\begin{array}{l}\text { Discriminant oil } \\
\text { constituents (set } 1 \text { ) }\end{array}$ & $\begin{array}{l}\text { Canonical variate } \\
\text { V1 }\end{array}$ & $\begin{array}{l}\text { Edapho-climactic factors } \\
\text { (set } 2)\end{array}$ & $\begin{array}{c}\text { Canonical variate } \\
\text { W1 }\end{array}$ \\
\hline (E)-Caryophyllene & -0.7452 & Clay / (\%) & 0.8807 \\
\hline$\alpha$-Guaiene & -0.3039 & $\mathrm{P} /\left(\mathrm{mg} \mathrm{dm}^{-3}\right)$ & 0.8807 \\
\hline Germacrene D & -0.1161 & $\mathrm{~K} /\left(\mathrm{mg} \mathrm{dm}^{-3}\right)$ & 0.8807 \\
\hline Bicyclogermacrene & -0.1248 & $\mathrm{~S} /\left(\mathrm{mg} \mathrm{dm}^{-3}\right)$ & 0.8807 \\
\hline$\delta$-Cadinene & -0.2408 & $\mathrm{Cu} /\left(\mathrm{mg} \mathrm{dm}^{-3}\right)$ & 0.8807 \\
\hline Ledol & 0.8503 & $\mathrm{Mn} /\left(\mathrm{mg} \mathrm{dm}^{-3}\right)$ & -0.8807 \\
\hline Spathulenol & 0.2336 & $\mathrm{Zn} /\left(\mathrm{mg} \mathrm{dm}^{-3}\right)$ & 0.8807 \\
\hline Globulol & 0.2303 & Base saturation / (\%) & 0.8807 \\
\hline \multirow[t]{4}{*}{ Guaiol } & 0.6321 & Al saturation / (\%) & -0.8807 \\
\hline & & Temperature / $\left({ }^{\circ} \mathrm{C}\right)$ & -0.2885 \\
\hline & & Precipitation / (mm) & -0.3745 \\
\hline & & Soil humidity / (\%) & -0.3114 \\
\hline Eingenvalue & & & 0.6555 \\
\hline Canonical correlation & & & 0.8097 \\
\hline Wilks' lambda & & & 0.0833 \\
\hline Bartlett's $C h i$-square & & & 151.62 \\
\hline Degrees of freedom & & & 108 \\
\hline$P$-value & & & 0.0036 \\
\hline \multicolumn{4}{|c|}{ Cumulative variance / $(\%)$ : } \\
\hline of discriminant oil & & & 14.31 \\
\hline of discriminant oi & imactic relation & & 39.89 \\
\hline
\end{tabular}

collected in Santa Catarina and Paraná states in South Brazil, in which essential oils were obtained by different methods, such as hydrodistillation by Clevenger apparatus, supercritical fluid extraction, and hexane extract. ${ }^{18}$

To judge from the current available data, a trend of oxidised monoterpenes seems to accompany the transition of sampling sites from the Brazilian Cerrado (present study) to sites in southern Brazil and Argentina. The same pattern is found when the climate is considered. Along this direction, a gradual change from the hot and dry to the cold and humid Austral Continental type occurs, ${ }^{30}$ suggesting that edapho-climactic variations may explain polymorphism in essential oils. A similar oxidative gradient was described for the emmotin sesquiterpenoids in South America, ${ }^{31}$ and for monoterpenes and sesquiterpenes in Hyptis suaveolens (Lamiaceae), but with an increase in the oxidised level from the Brazilian Cerrado to the Amazonian region. ${ }^{32}$

Thus, the variation pattern in the essential oil may reflect selective pressures in the different ecological and geographical environments (ecotypes) or indicate that the observed chemical variations could be due to the existence of chemotypes for $B$. trimera, which have not yet been described for Baccharis species. Therefore, caution is necessary in the use of carquejyl acetate as a chemomarker of $B$. trimera for this compound could be in part environmentally determined. In spite of the correlation obtained for the oil composition with edapho-climactic factors and the variance explained by the environmental data set (Table 2), there is an outstanding percentage of variability in oil composition that should be the subject of subsequent genetic studies.

\section{Experimental}

\section{Plant material}

B. trimera samples were collected between March 2003 and February 2004 in Lavras city (S 21 ${ }^{\circ} 13^{\prime}$; W $44^{\circ}$ 57'; 919 m), Minas Gerais State, Brazil, and were identified by Prof. Dr. Manoel Losada Gavilanes of Departamento de Biologia, Universidade Federal de Lavras (UFLA), Minas Gerais State, Brazil. Samples from their natural habitat were collected on a monthly basis, and plantlets obtained by cutting propagation were cultivated in an experimental field at the Setor de Horticultura of Universidade Federal de Lavras (UFLA), ${ }^{21}$ Minas Gerais State, Brazil. The natural habitat was located at $20 \mathrm{~km}$ from the cultivated samples. Field trial was performed in the form of a randomised block with three replications. In the March-May period, the plants included both leaves and flowers. Voucher specimen is deposited in UFLA's Herbarium (code number 169933).

In order to assess the chemical composition of oils, the aerial parts of 40-100 randomised individual plants representing the local population as homogeneous samples 
- were simultaneously collected from cultivated plants and from their original natural habitat and dried by dehumidification in Arsec 160 apparatus for 3 days at $35^{\circ} \mathrm{C}$ until constant weight. After having been chopped, the dried phytomass $(50 \mathrm{~g}$ ) was submitted to hydrodistillation $(1 \mathrm{~h})$ using a modified Clevenger-type apparatus. At the end of each distillation, oils were collected by extracting the aqueous solution with $\mathrm{CH}_{2} \mathrm{Cl}_{2}$ $(3 \times 90 \mathrm{~mL})$, drying the dichloromethane layer with anhydrous $\mathrm{MgSO}_{4}$, and removing the solvent in a rotavapor under reduced pressure at $40{ }^{\circ} \mathrm{C}$. The oils were transferred to glass flasks and kept at a temperature of $-18{ }^{\circ} \mathrm{C}$. Oil yields (\%) were based on the dried weight of plant samples. All experiments were conducted in triplicates.

\section{Soil analyses}

Five-soil samples were collected at a $20 \mathrm{~cm}$ depth in each locality in March 2003. These samples were then pooled together to form one composite sample and subsequently air-dried, thoroughly mixed, and sieved (2 $\mathrm{mm})$. The portion which was finer than $2 \mathrm{~mm}$ was kept for physical and chemical analysis. ${ }^{33}$ The $\mathrm{pH}$ was determined in a 1:1 soil-water volume ratio. $\mathrm{Ca}, \mathrm{Mg}$ and $\mathrm{Al}$ were extracted with $1 \mathrm{~mol} \mathrm{~L}^{-1} \mathrm{KCl}$, and $\mathrm{P}, \mathrm{K}, \mathrm{Zn}, \mathrm{Cu}$, $\mathrm{Fe}, \mathrm{Mn}$ were extracted with Mehlich's solution. Organic matter, cationic exchange capacity (CEC), potential acidity $(\mathrm{H}+\mathrm{Al})$, base saturation, $\mathrm{Al}$ saturation, and soil texture were determined by applying the usual methods. ${ }^{33}$ Soil humidity (at a $20 \mathrm{~cm}$ depth) was determined monthly by the gravimetric method, and was conducted in triplicates. Mean monthly values of temperature (minimal, average and maximal), precipitation, and humidity were obtained from UFLA's climatological station. Environmental factor data originating from both climatological records and soil analysis were ordered in an edapho-climactic matrix with 26 variables for each sampling site. In both data sets, essential oil components and edapho-climactic characteristics, a canonical correlation procedure was applied. This method finds the linear combinations of the variables which have the highest correlation between them.

In geographical terms, the natural habitat presents a loam texture, while the cultivated field has a sandy loam. Site elevation, mean annual rainfall, mean temperature, and mean annual relative humidity are, however, similar.

\section{Chemical analysis}

Oil sample analyses were performed on a GC-MS Shimadzu QP5050A instrument under the following conditions: a CBP-5 (Shimadzu) fused silica capillary column $(30 \mathrm{~m} \times 0.25 \mathrm{~mm}$ i.d., $0.25 \mu \mathrm{m}$ film thickness $)$ connected to a quadrupole detector operating in the EI mode at $70 \mathrm{eV}$ with a scan mass range of $40-400 \mathrm{~m} / \mathrm{z}$ at a sampling rate of 1.0 scan s$^{-1}$; carrier gas: $\mathrm{He}\left(1 \mathrm{~mL} \mathrm{~min}^{-1}\right)$; injector and interface temperatures of $220{ }^{\circ} \mathrm{C}$ and 240 ${ }^{\circ} \mathrm{C}$, respectively, with a split ratio of 1:20. The injection volume was $0.2 \mu \mathrm{L}\left(20 \%\right.$ in $\left.\mathrm{CH}_{2} \mathrm{Cl}_{2}\right)$ and the oven temperature was raised from $60{ }^{\circ} \mathrm{C}$ to $246{ }^{\circ} \mathrm{C}$, with an increase of $3{ }^{\circ} \mathrm{C} \mathrm{min}^{-1}$, then $10^{\circ} \mathrm{C} \mathrm{min}^{-1}$ to $270{ }^{\circ} \mathrm{C}$, holding the final temperature for $5 \mathrm{~min}$. Individual components were identified by comparing their Kovats retention indexes (RI), ${ }^{34}$ made by a co-injection with a $\mathrm{C}_{8}-\mathrm{C}_{32}$ $n$-alkanes series, ${ }^{35}$ mass spectra with those of the literature, and a computerised MS-data base using NIST libraries. ${ }^{34,36}$

\section{Chemical variability}

Principal Component Analysis (PCA) was applied in order to examine the interrelationships between populations and their essential oil constituents using Système Portable d'Analyse des Données Numériques-SPAD.N software package, version 2.5, Centre International de Statistique et d'Informatique Appliquées, France (1994). Cluster analysis was also applied to the study of similarity of samples on the basis of essential oil constituent distribution. Nearest neighbour complete linkage technique by Benzécri algorithm was used as an index of similarity, and hierarchical clustering was performed according to the Ward's variance minimizing method. ${ }^{27,37}$

Canonical discriminant analysis using SAS CANDISC procedure (Statistical Analysis System, SAS Institute Inc., Cary, NC, 1996) was used to differentiate between populations and clusters on the basis of oil composition. The predictive ability of canonical discriminant functions was evaluated by cross-validation leaving one group approach as implemented in SAS statistical package.

Oil variability and edapho-climactic factors relationships were obtained by a canonical correlation analysis implemented using the SAS CANCORR procedure. The predictive ability was evaluated by canonical redundancy analysis with a standardised variance coefficient.

Average multiple comparisons were established by the Scott-Knott test by ANOVA. $P$-values less than 0.05 were considered to be significant.

\section{Acknowledgments}

The authors are indebted to CNPq, PADCT III and FUNAPE/UFG for financial support, CNPq for fellowship to F.G.S., and PIBIC (C.B.A.O.). Thanks are also 
expressed to Dr. Manoel L. Gavilanes of Departamento de Biologia-UFLA for the botanical identification, and the anonymous referees for the valuable suggestions which improved this manuscript.

\section{Supplementary Information}

Supplementary data are available free of charge at http://jbcs.sbq.org.br, as PDF file.

\section{References}

1. Verdi, L. G.; Brighente, I. M. C.; Pizzolatti, M. G.; Quim. Nova 2005, 28, 85; Jarvis, B. B.; Wang, S.; Cox, C.; Rao, M. M.; Philip, V.; Varaschin, M. S.; Barros, C. S.; Nat. Tox. 1996, 4, 58; Rozza, D. B.; Raymundo, D. L.; Corrêa, A. M. R.; Leal, J.; Seitz, A. L.; Driemeier, D.; Colodel, E. M.; Pesq. Vet. Bras. 2006, 26, 21.

2. Corrêa, M. P.; Dicionário das Plantas Úteis do Brasil e das Exóticas Cultivadas, Instituto Brasileiro de Desenvolvimento Florestal: Rio de Janeiro, 1984.

3. Souza, M. P.; Matos, M. E. O.; Matos, F. J. A.; Machado, M. I. C.; Craveiro, A. A.; Constituintes Químicos Ativos de Plantas Medicinais Brasileiras, Edições UFC: Fortaleza, 1991.

4. Gene, R. M.; Cartaña, C.; Adzet, T.; Marlin, E.; Parella, T.; Cañigueral, S.; Planta Med. 1996, 62, 232.

5. Soicke, H.; Leng-Peschlow, E.; Planta Med. 1987, 1, 37.

6. Lapa, A. J.; Fischman, L. A.; Gamberini, M. T.; Abstracts of the $1^{\text {st }}$ International Symposium on Natural Drugs and Digestive Tract, Nápolis, Italy, 1992; Oliveira, A. C. P.; Endringer, D. C.; Amorim, L. A. S.; Graças, M.; Brandão, M. D. G.; Coelho, M. M.; J. Ethnopharmacol. 2005, 102, 465; Coelho, M. G. P.; Reis, P. A.; Gava, V. B.; Marques, P. R.; Gayer, C. R.; Laranja, G. A. T.; Felzenswalb, I.; Sabino, K. C. C.; Toxicol. Lett. 2004, 154, 69.

7. Torres, L. M. B.; Gamberini, M. T.; Roque, N. F.; Lima-Landman, M. T.; Souccar, C.; Lapa, A. J.; Phytochemistry 2000, 55, 617.

8. Januario, A. H.; Santos, S. L.; Marcussi, S.; Mazzi, I. V.; Pietro, R. C. L. R.; Sato, D. N.; Ellena, J.; Sampaio, S. V.; Franca, S. C.; Soares, A. N.; Chem.-Biol. Interact. 2004, 150, 243; Nakasugi, T.; Komai, K.; J. Agric. Food Chem. 1998, 46, 2560; Abad, M. J.; Bermejo, P.; Gonzales, E.; Iglesias, I.; Irurzun, A.; Carrasco, L.; Gen. Pharmacol. 1999, 32, 499; Avancini, C. A. M.; Wiest, J. M.; Mundstock, E.; Arq. Bras. Med. Vet. Zootec. 2000, 52, 230; Oliveira, S. Q.; Trentin, V. H.; Kappel, V. D.; Barelli, C.; Gosmann, G.; Reginatto, F. H.; Pharm. Biol. 2005, 43, 434; Luize, P. S.; Tiuman, T. S.; Morello, L. G.; Maza, P. K.; Ueda-Nakamura, T.; Dias Filho, B. P.; Cortez, D. A. G.; Mello, J. C. P.; Nakamura, C. V.; Rev. Bras. Cienc. Farm. 2005, 41, 85.

9. Boldt, P. E.; Baccharis (Asteraceae). A Review of its Taxonomy, Phytochemistry, Ecology, Economic Status, Natural Enemies and the Potential for its Biological Control in the United States; The Texas A \& M University System: Texas, 1989.
10. Naves, Y. R.; Bull. Soc. Chim. Fr. 1959, 292, 1871.

11. Naves, Y. R.; Helv. Chim. Acta 1959, 42, 1996.

12. Dolejs, L. V.; Herout, V.; Sorm, F.; Collect. Czech. Chem. Commun. 1961, 26, 811.

13. Thomas, A. F.; Helv. Chim. Acta 1967, 50, 963.

14. Bauer, L.; Silva, G. B.; Siqueira, N. C. S.; Bach, C. T. M.; Sant'Ana, B. M. S.; Rev. Cent. Ciênc. Saúde 1978, 6, 7.

15. Siqueira, N. C. S.; Silva, G. A. A. B.; Alice, C. B.; Nitschke, M.; Rev. Bras. Farm. 1985, 1, 36.

16. Chialva, F.; Doglia, G.; J. Essent. Oil Res. 1990, 2, 173.

17. Simões-Pires, C. A.; Debenedetti, S.; Spegazzini, E.; Mentz, L. A.; Matzenbacher, N. I.; Limberger, R. P.; Henriques, A. T.; Pl. Syst. Evol. 2005, 253, 23.

18. Poça, A. M. P. C.; M. Sc. Dissertation, Universidade Federal do Paraná, Brazil, 2005; Silva, D. C. M. N.; M. Sc. Dissertation, Universidade Federal de Santa Catarina, Brazil, 2004; Carvalho, R. I. N.; Cardon, L. M.; Jaremtchuk, C. C.; Kanawate, E. N.; Silva, J. E. C.; Carqueja e Espinheira-Santa na Região Metropolitana de Curitiba: da Produção ao Comércio, Life Serviços Gráficos: Curitiba, 2003.

19. Silva, S. R.; Buitron, X.; Oliveira, L. H.; Martins, M. V. M.; Plantas Medicinais do Brasil: Aspectos sobre Legislação e Comércio, IBAMA, 2001; Steenbock, W. In Productos Forestales, Medios de Subsistencia y Conservación; Brower, J., ed.; Centro para la Investigación Forestal Internacional: Indonésia, 2004, ch. 12.; SEAB (Secretaria de Estado da Agricultura e do Abastecimento do Paraná), Departamento de Economia Rural, Divisão das Estatísticas Básicas, 2004.

20. http://www.genamaz.org.br/estudcosmetico01.html, accessed in December 2006.

21. Bona, C. M.; Biasi, L. A.; Costa, G.; Zanette, F.; Nakashima, T.; Rev. Bras. Pl. Med. 2003, 6, 28.

22. Azevedo, N. R.; Campos, I. F. P.; Ferreira, H. D.; Portes, T. A.; Santos, S. C.; Seraphin, J. C.; Paula, J. R.; Ferri, P. H.; Phytochemistry 2001, 57, 733.

23. Corrêa Júnior, C.; Ming, L. C.; Scheffer, M. C.; Cultivo de Plantas Medicinais, Aromáticas e Condimentares, EMATER: Curitiba, 1991.

24. Nascimento, V. E.; Silva, F. G.; Pinto, J. E. B. P.; Sales, J. F.; Bertolucci, S. K. V.; Abstracts of the Congresso de Cultura de Tecidos, Lavras: Brazil, 2003.

25. Silva, F. G.; Pinto, J. E. B. P.; Sales, J. F.; Divino, S. P.; Bertolucci, S. K.; Cienc. Agrotecnol. 2003, 27, 535.

26. Ferracini, V. L.; Paraiba, L. C.; Filho, H. F.; Silva, A. G.; Nascimento, L. R.; Marsaioli, A. J.; Planta Med. 1995, 7, 355; Zunino, M. P.; Newton, M. N.; Maestri, D. M.; Zygadlo, J. A.; Planta Med. 1998, 64, 86.

27. Ward, J. H.; J. Am. Stat. Assoc. 1963, 58, 238.

28. Wold, A.; Eriksson, L. In Chemometric Methods in Molecular Design; Waterbeemd, H., ed.; VCH: Weinheim, 1995, vol. 2, ch. 5 . 
29. Sturgeon, K. B.; Evolution 1979, 33, 803; Langenheim, J. H.; J. Chem. Ecol. 1994, 20, 1223; Figueiredo, A. C.; Barroso, J. G.; Pedro, L. G.; Scheffer, J. J. C. In Essential Oils: Basic and Applied Research; Franz, C.; Mathe, A.; Buchbauer, G., eds.; Allured: Illinois, 1997; Robles, C.; Garzino, S.; Phytochemistry 2000, 53, 71.

30. Azevedo, L. G.; Caser, R. L.; Abstract of the Quinto Simpósio sobre o Cerrado, Brasília, Brazil, 1980.

31. Kaplan, M. A. C.; Ribeiro, J.; Gottlieb, O. R.; Phytochemistry 1991, 30, 2671.

32. Oliveira, M. J.; Campos, I. F. P.; Oliveira, C. B. A.; Santos, M. R.; Souza, P. S.; Santos, S. C.; Seraphin, J. C.; Ferri, P. H.; Biochem. Syst. Ecol. 2005, 33, 275.
33. Silva, S. C.; Manual de Análises Químicas de Solos, Plantas e Fertilizantes, $1^{\text {a }}$ ed., Embrapa: Brasília, 1999.

34. Adams, R. P.; Identification of Essential Oil Components by Gas Chromatography/Quadrupole Mass Spectroscopy, Allured: Illinois, 2001.

35. van Den Dool, H.; Kratz, P. D. J. A.; J. Chromatogr. 1963, 11, 463.

36. National Institute of Standards and Technology; PC version of the NIST/EPA/NIH Mass Spectral Database, U.S. Department of Commerce: Gaithersburg, 1998.

37. Benzécri, J. P.; L'Analyse des Données: la Taxinomie, Tome 1, Dunod: Paris, 1980.
Received: December 5, 2006

Web Release Date: August 9, 2007 


\section{Seasonal Variability in the Essential Oils of Wild and Cultivated Baccharis trimera}

\section{Fabiano G. Silva, ${ }^{a}$ Carolina B. A. Oliveira, ${ }^{b}$ José Eduardo B. P. Pinto, ${ }^{c}$ Vivian E. Nascimento, ${ }^{c}$} Suzana C. Santos, ${ }^{b}$ José C. Seraphin ${ }^{d}$ and Pedro H. Ferri ${ }^{*, b}$

${ }^{a}$ Laboratório de Cultura de Tecidos, Centro Federal de Educação Tecnológica de Rio Verde, CP 66, 75900-000 Rio Verde-GO, Brazil

${ }^{b}$ Laboratório de Bioatividade Molecular, Instituto de Química, Universidade Federal de Goiás, CP 131, 74001-970 Goiânia-GO, Brazil

${ }^{c}$ Laboratório de Cultura de Tecidos e Plantas Medicinais, Departamento de Agricultura, Universidade Federal de Lavras, CP 37, 37200-000 Lavras-MG, Brazil

${ }^{d}$ Núcleo de Estatística Aplicada, Instituto de Matemática e Estatística, Universidade Federal de Goiás, CP 131, 74001-970 Goiânia- GO, Brazil
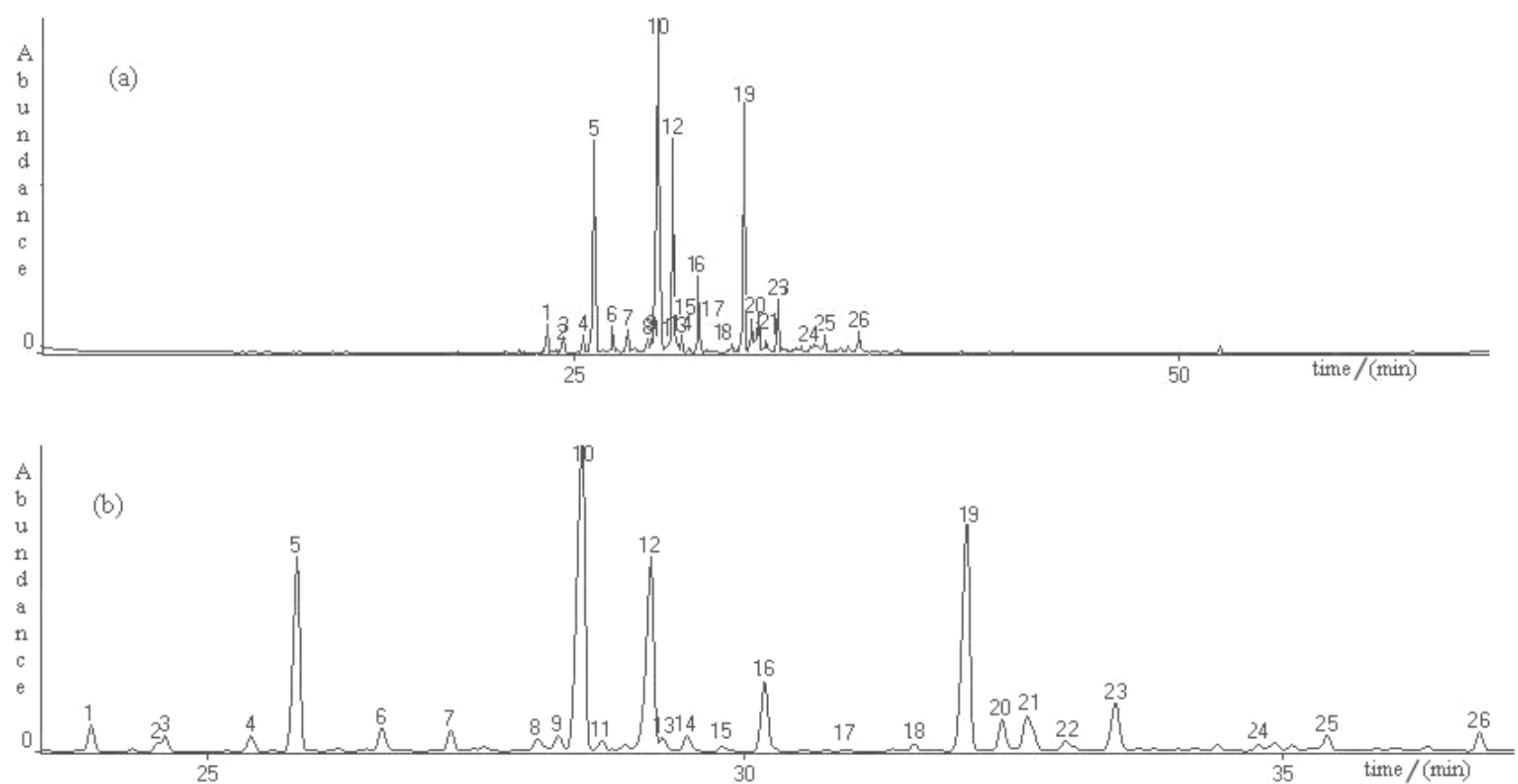

Figure S1. (a) Total Ion Count (TIC) obtained by GCMS of carqueja essential oil (Baccharis trimera); (b) TIC zoom showed the constituents (peak number) arranged in order of elution.

*e-mail: pedro@quimica.ufg.br 


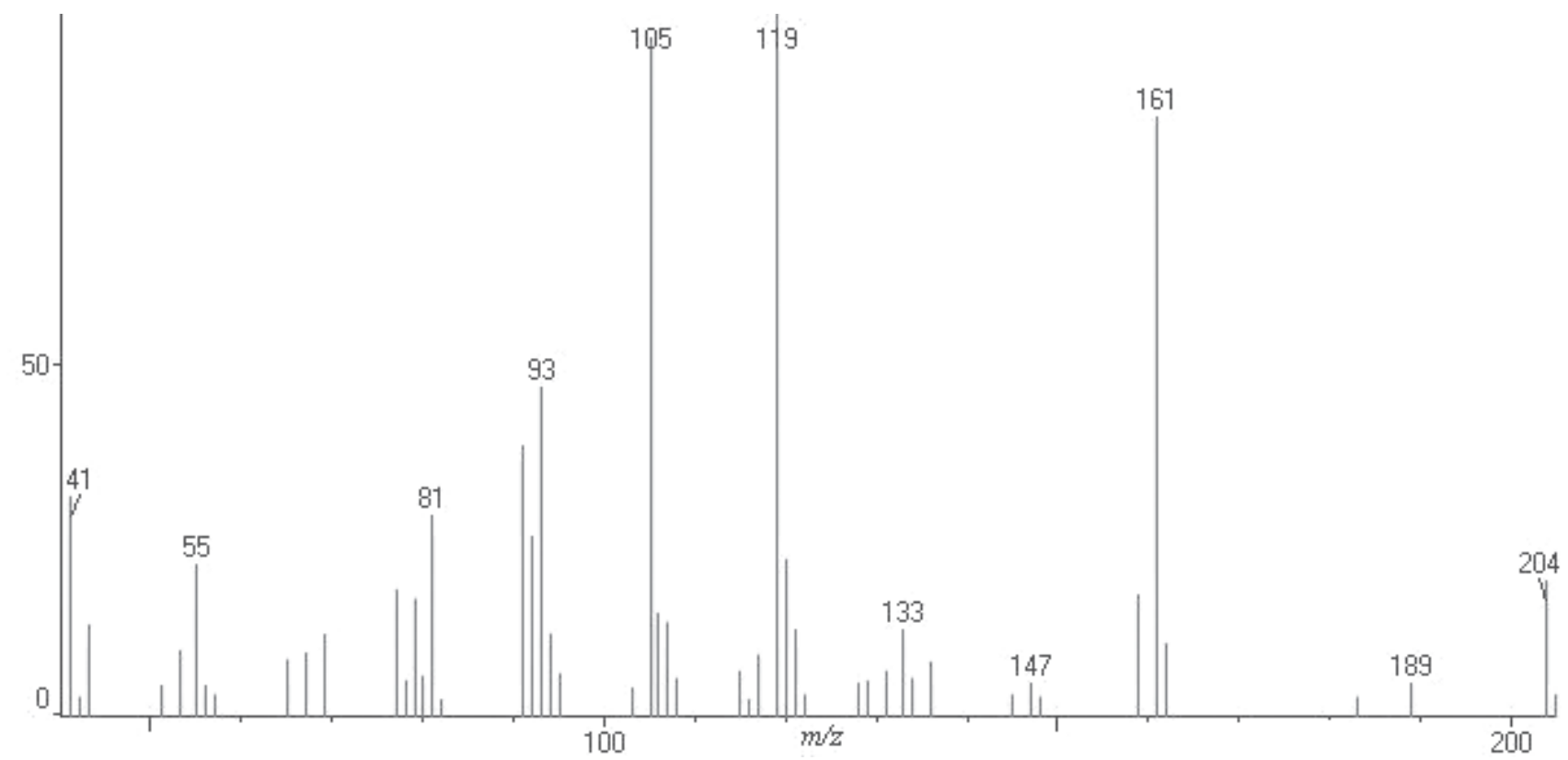

Figure S2. Mass spectrum of $\alpha$-copaene (peak 1).

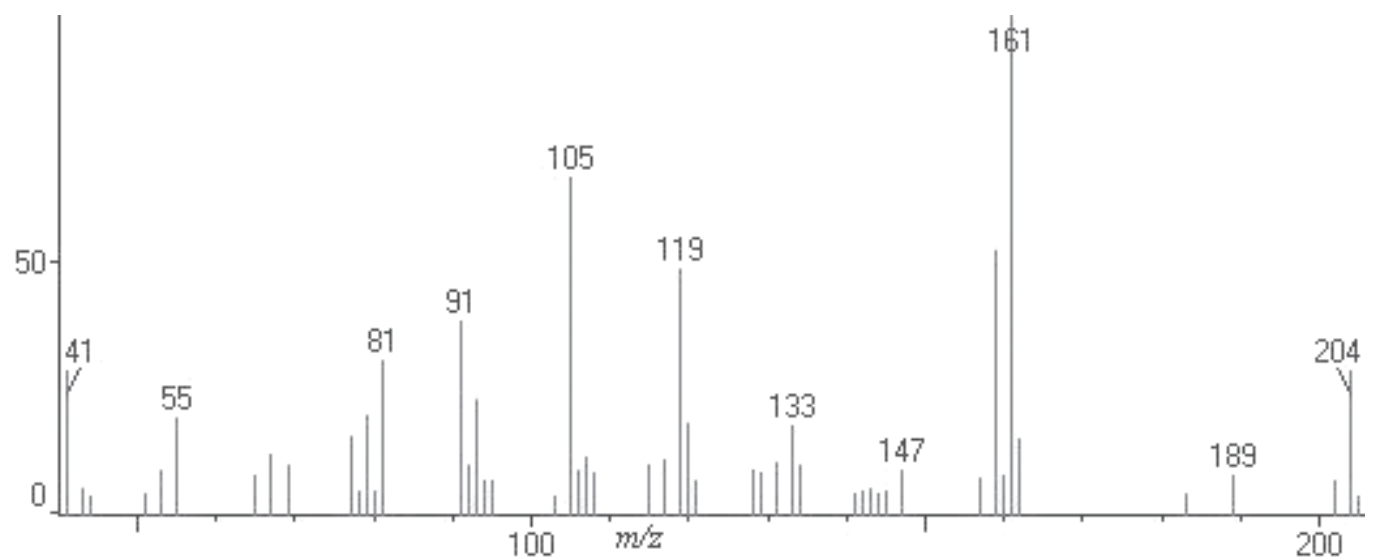

Figure S3. Mass spectrum of $\beta$-cubebene (peak 2).

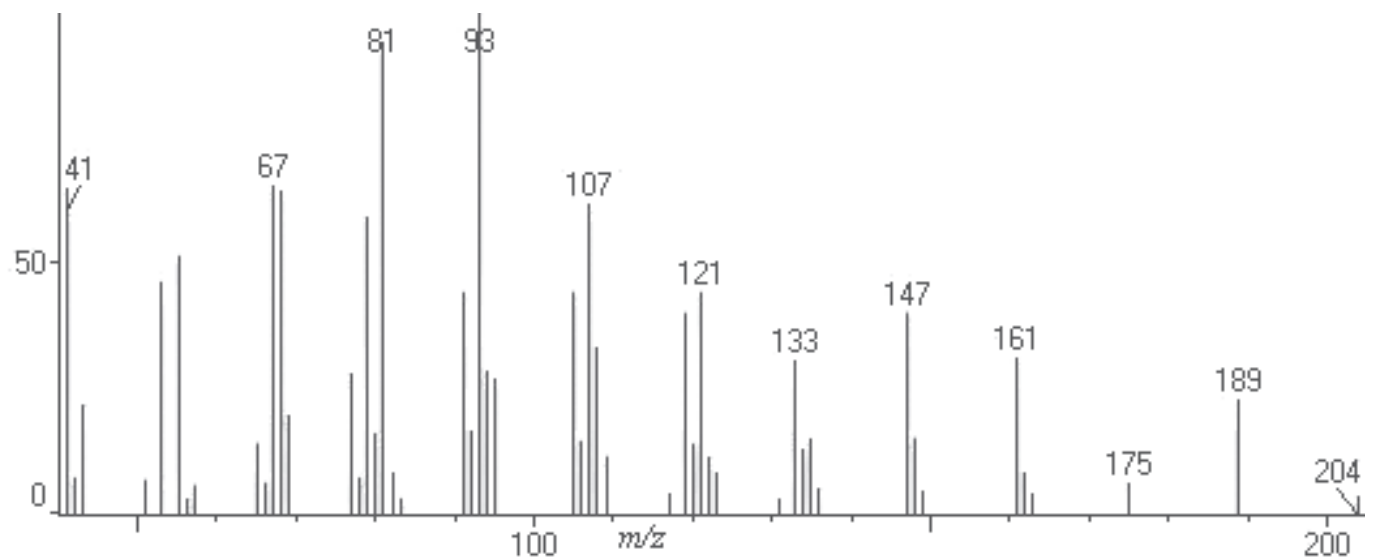

Figure S4. Mass spectrum of $\beta$-elemene (peak 3). 


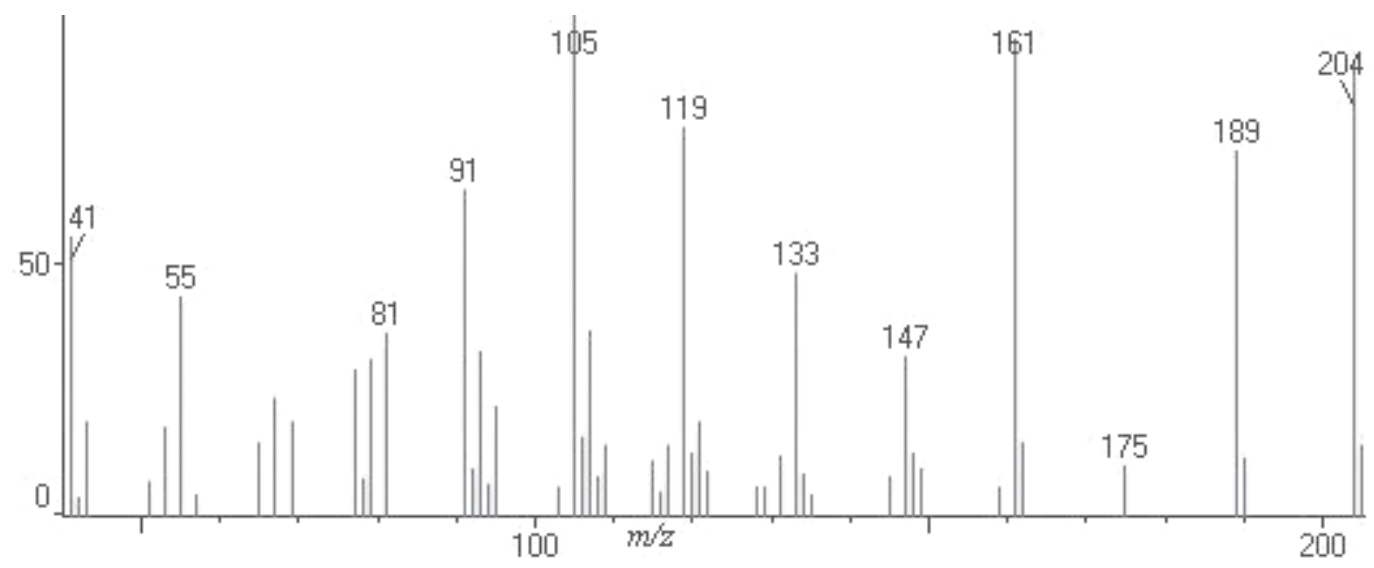

Figure S5. Mass spectrum of $\alpha$-gurjunene (peak 4).

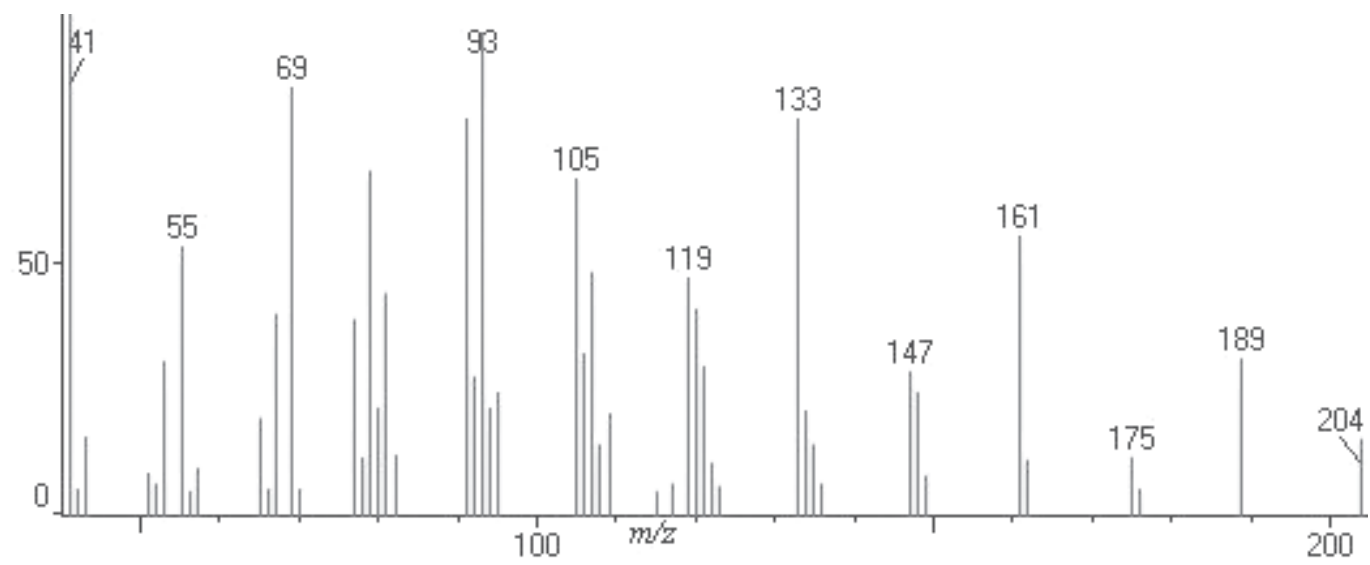

Figure S6. Mass spectrum of (E)-caryophyllene (peak 5).

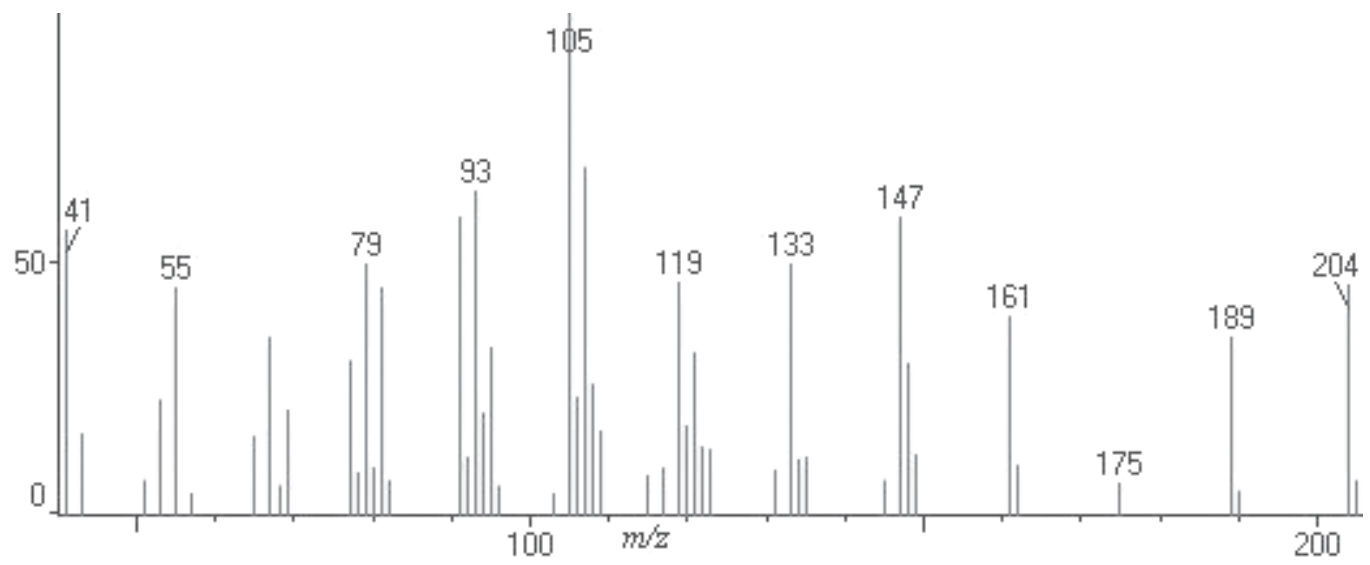

Figure S7. Mass spectrum of $\alpha$-guaiene (peak 6). 


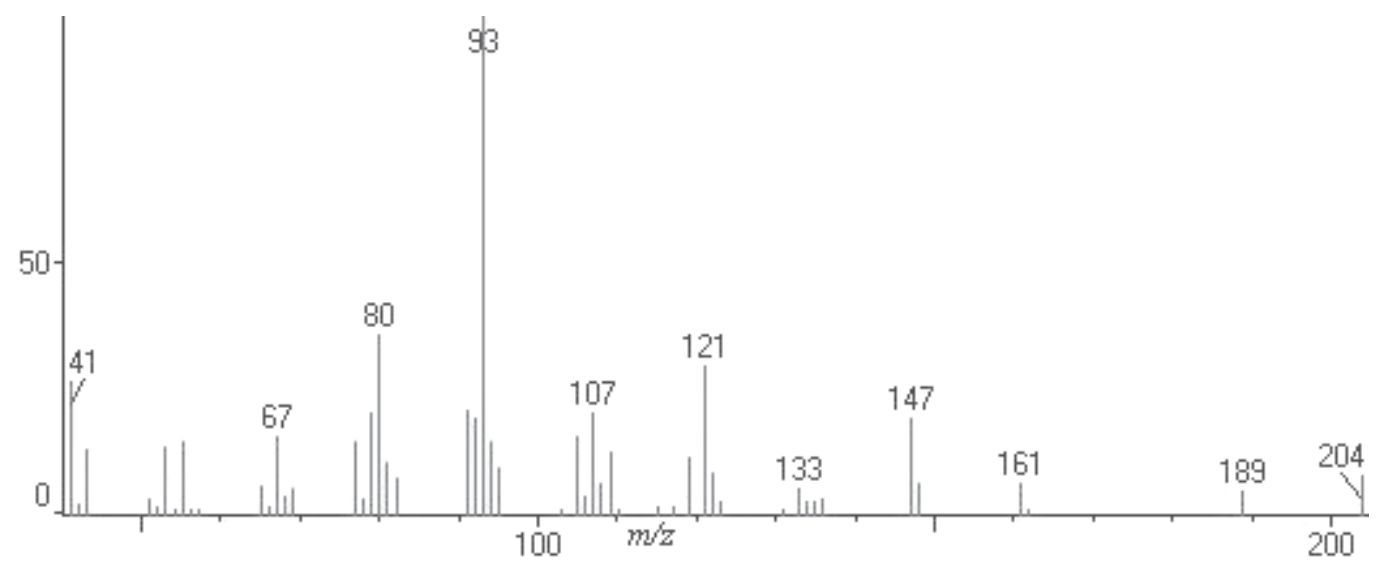

Figure S8. Mass spectrum of $\alpha$-humulene (peak 7).

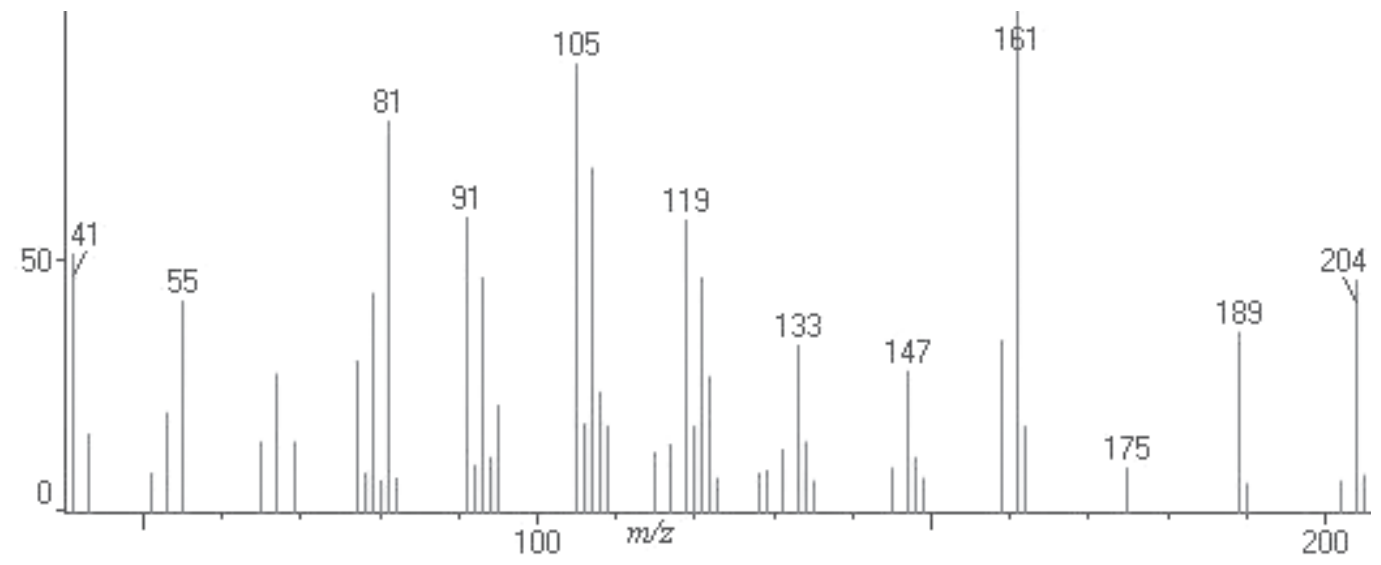

Figure S9. Mass spectrum of $\gamma$-gurjunene (peak 8).

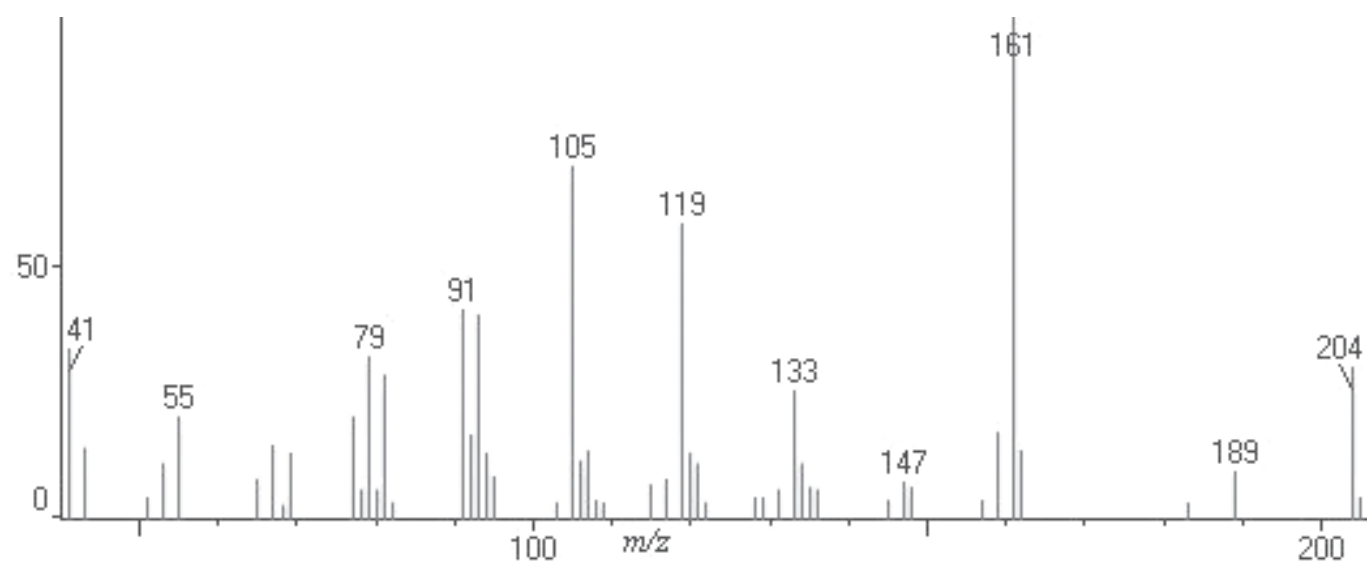

Figure S10. Mass spectrum of $\gamma$-muurolene (peak 9). 


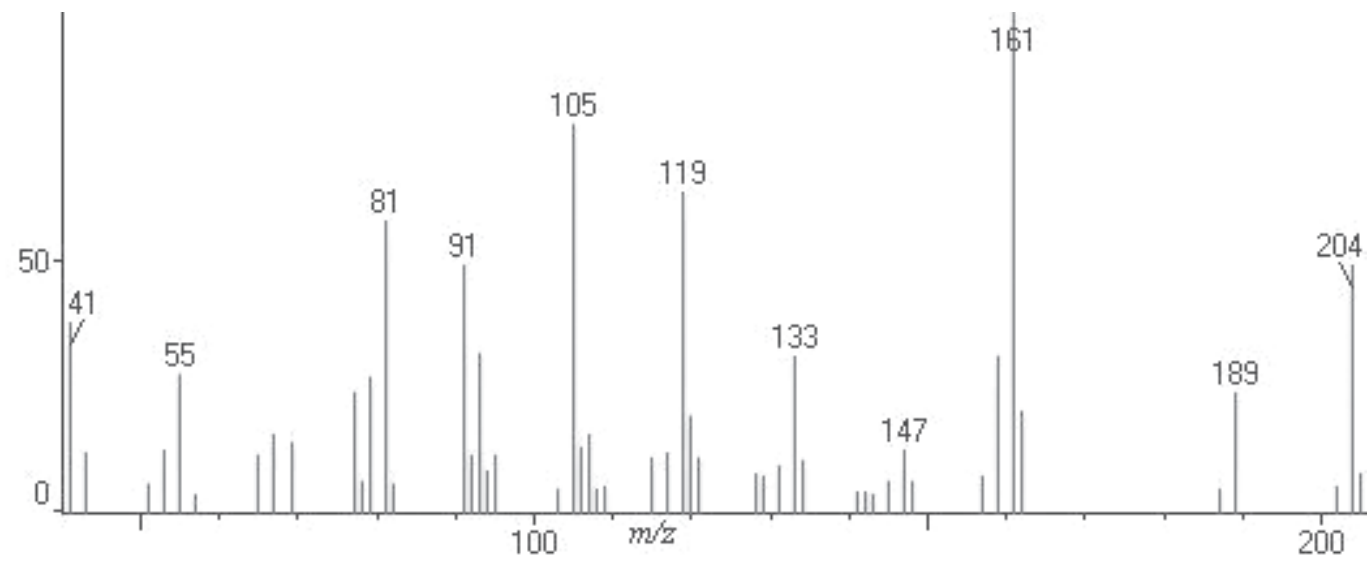

Figure S11. Mass spectrum of germacrene D (peak 10).

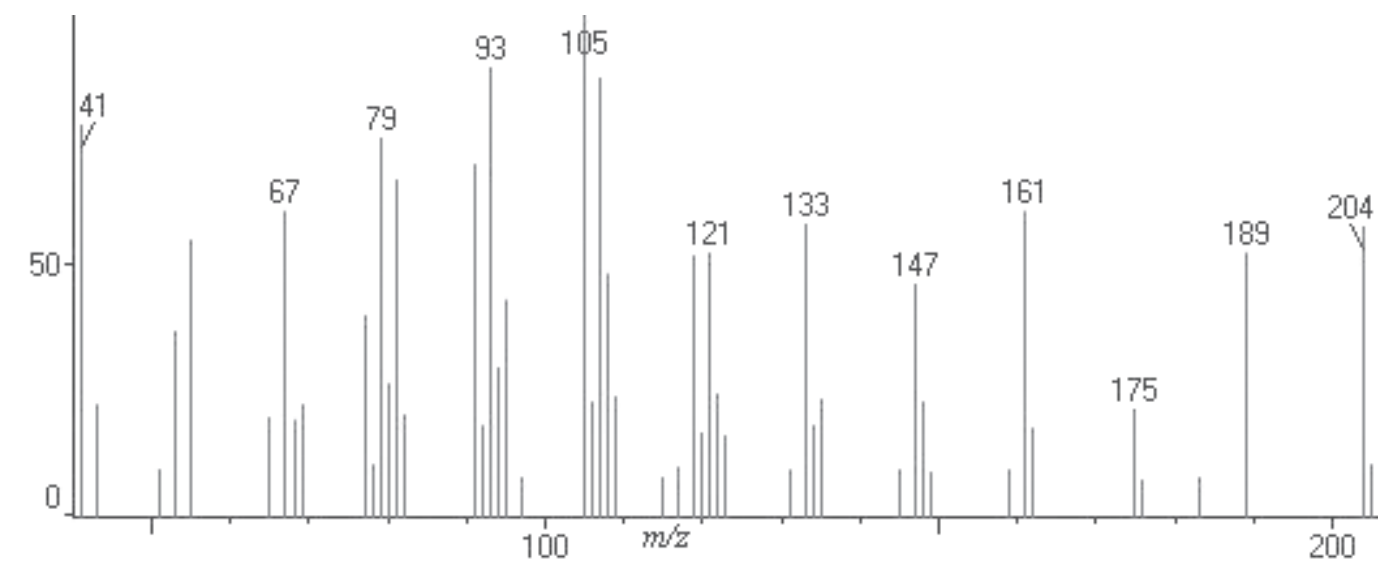

Figure S12. Mass spectrum of $\beta$-selinene (peak 11).

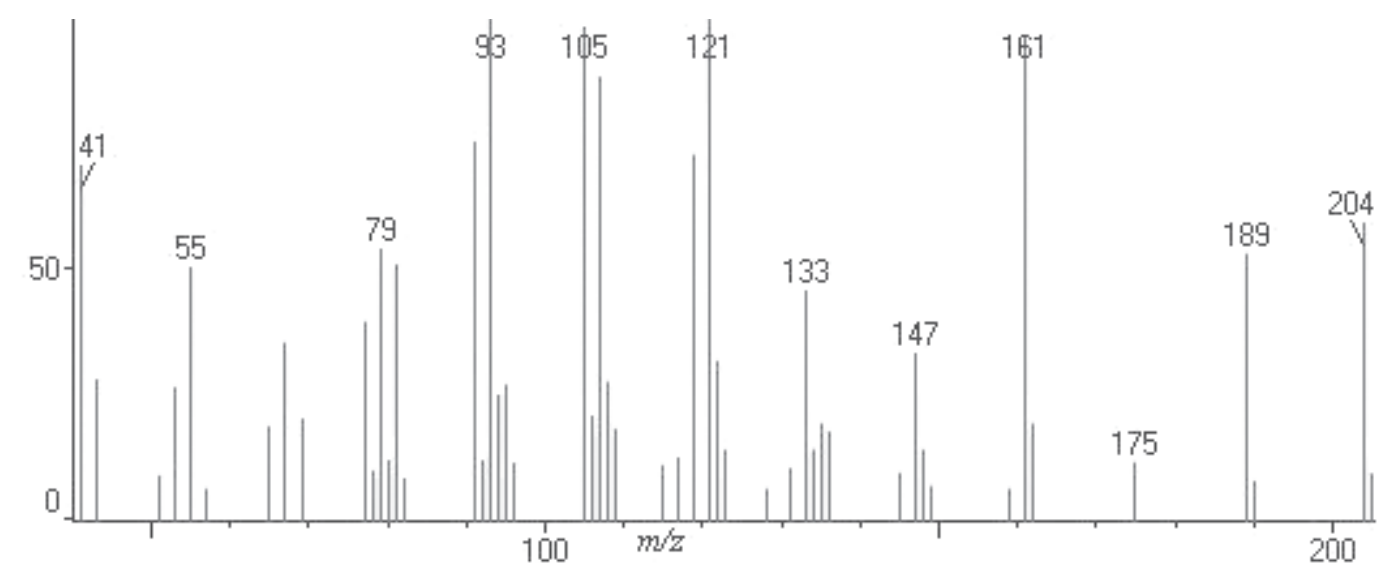

Figure S13. Mass spectrum of bicyclogermacrene (peak 12). 


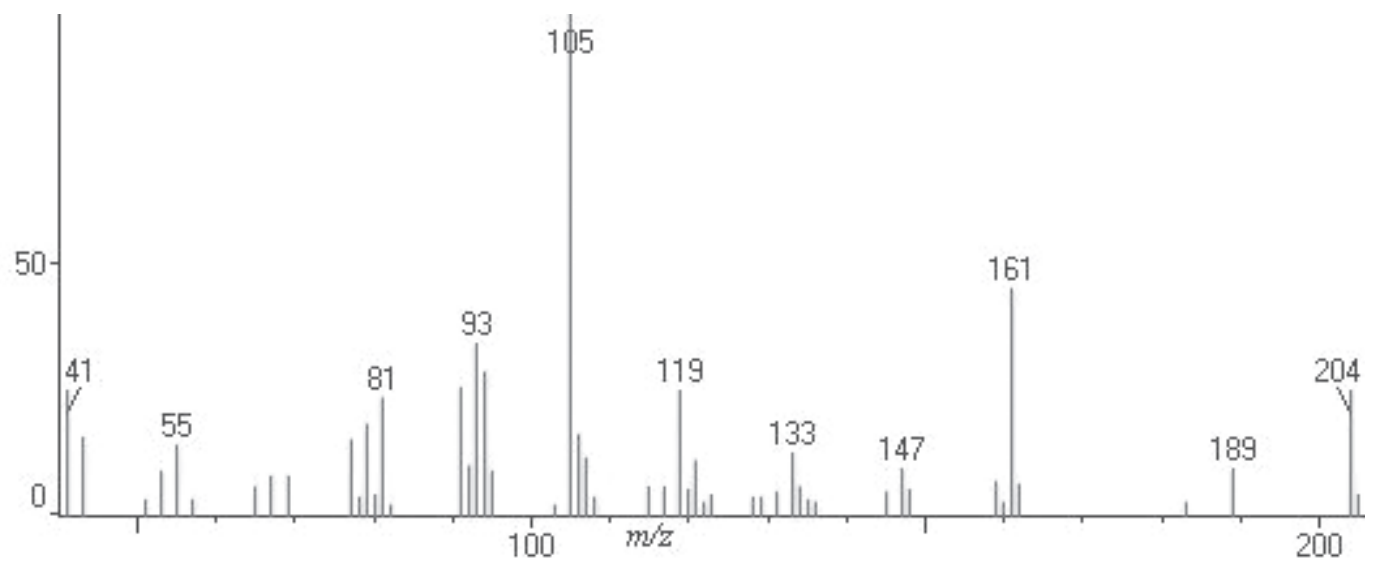

Figure S14. Mass spectrum of $\alpha$-muurulene (peak 13).

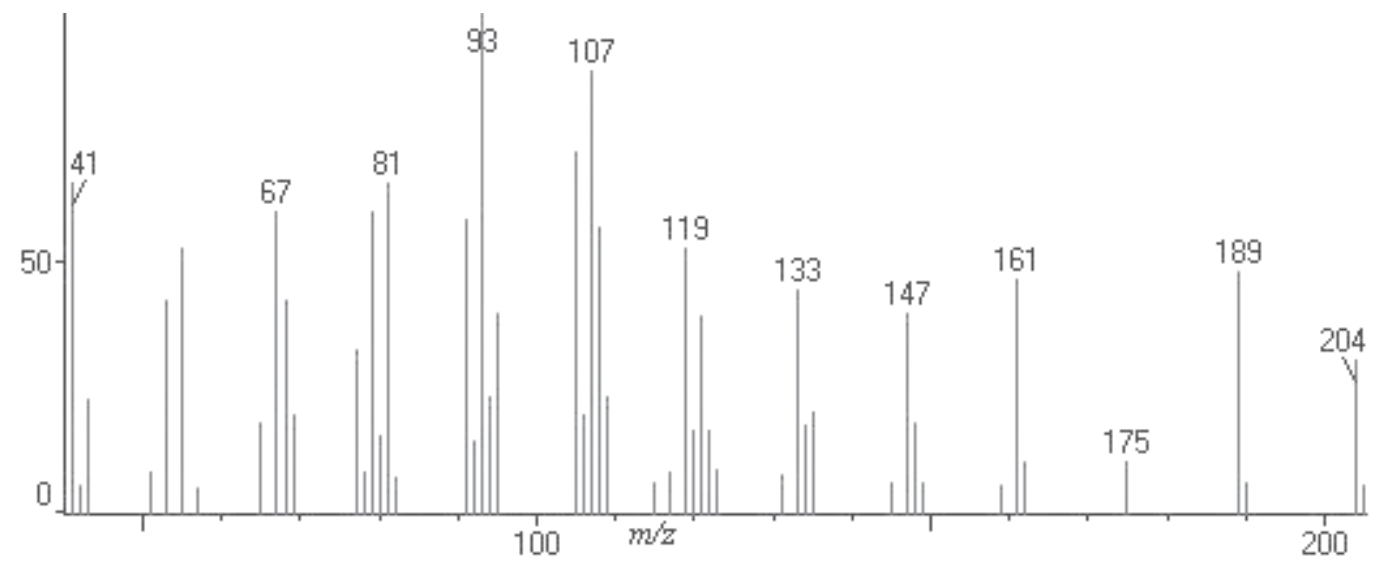

Figure S15. Mass spectrum of $\alpha$-bulnecene (peak 14).

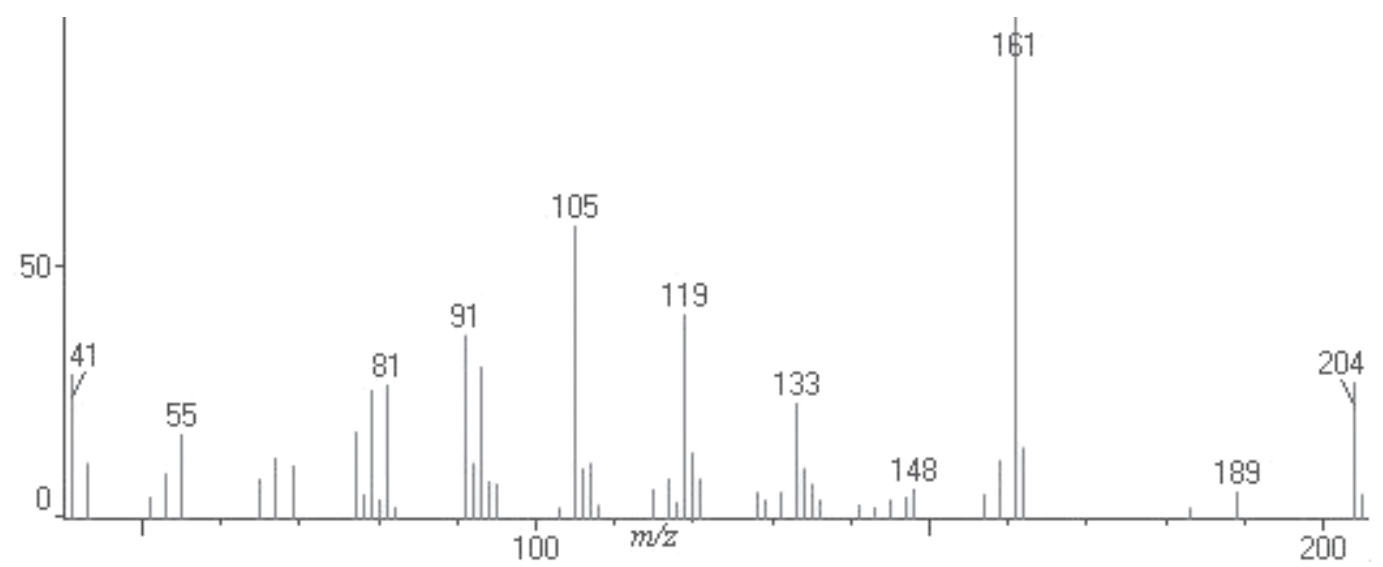

Figure S16. Mass spectrum of $\gamma$-cadinene (peak 15). 


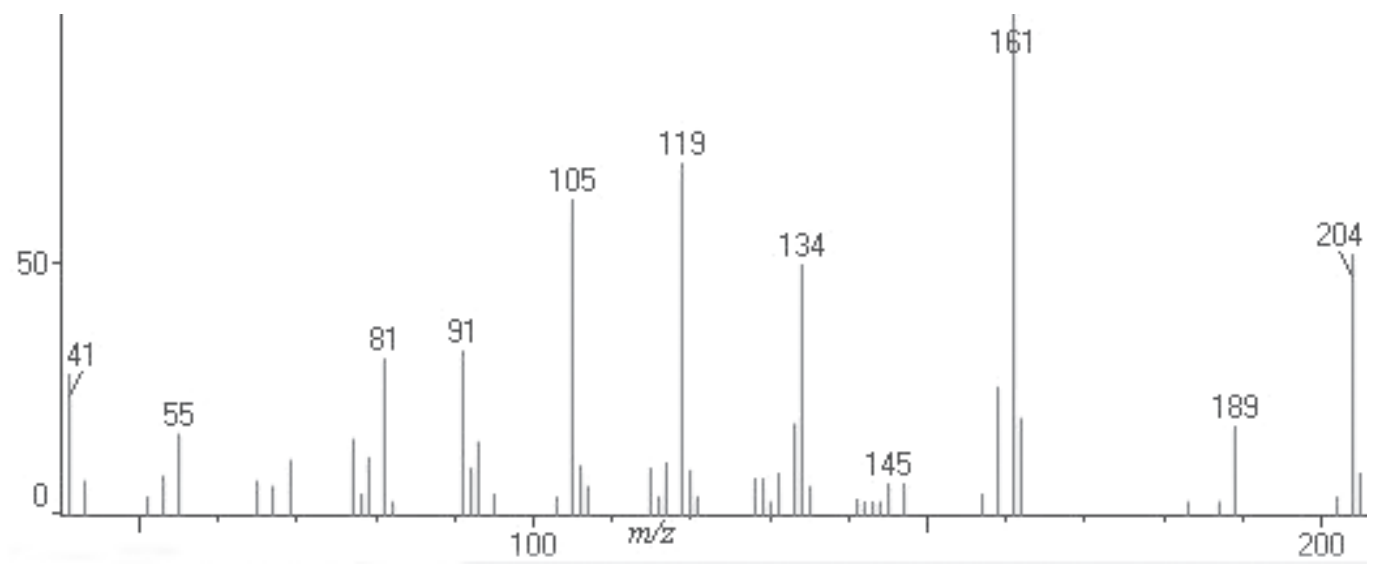

Figure S17. Mass spectrum of $\delta$-cadinene (peak 16).

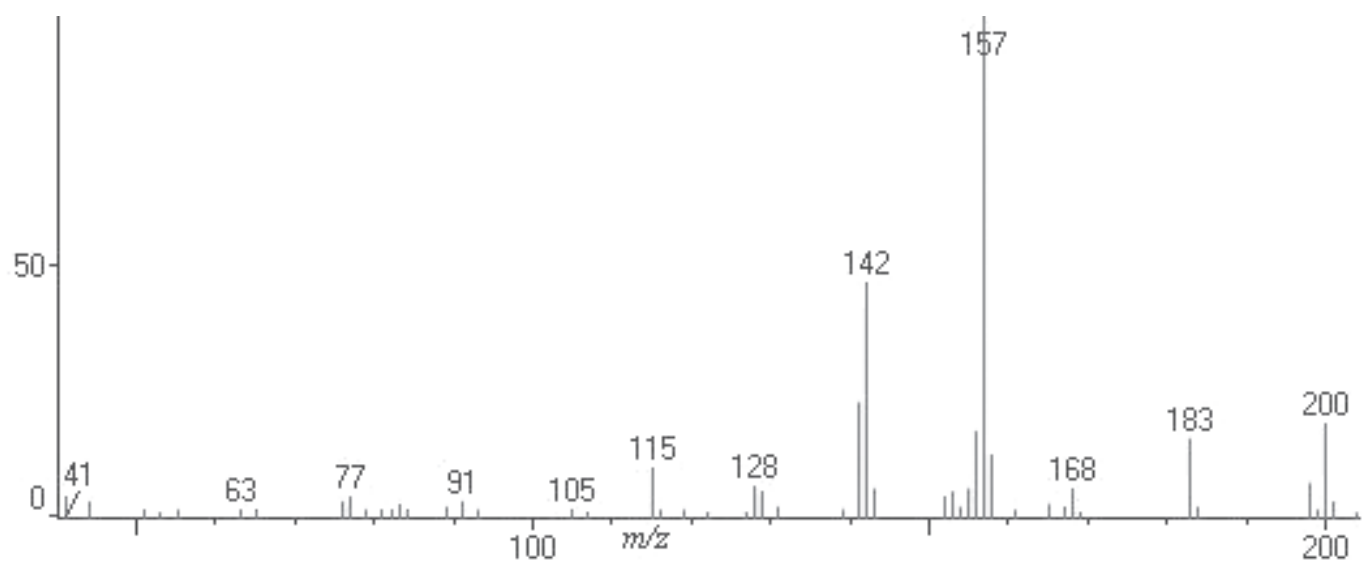

Figure S18. Mass spectrum of $\delta$-calacorene (peak 17).

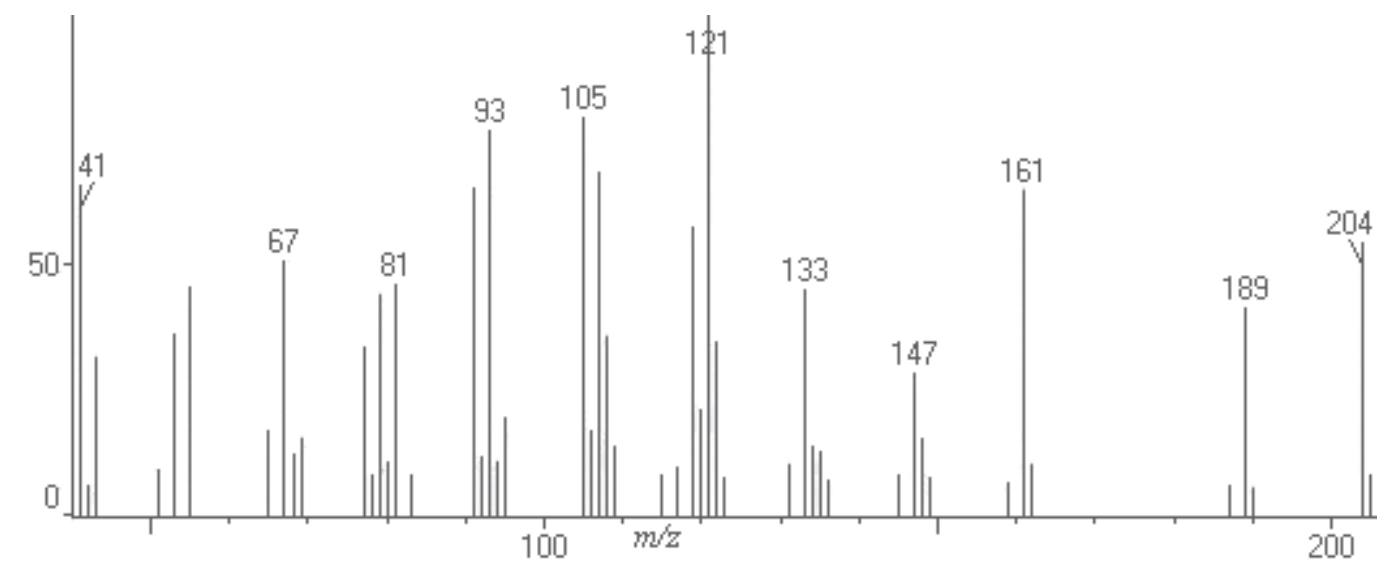

Figure S19. Mass spectrum of germacrene B (peak 18). 


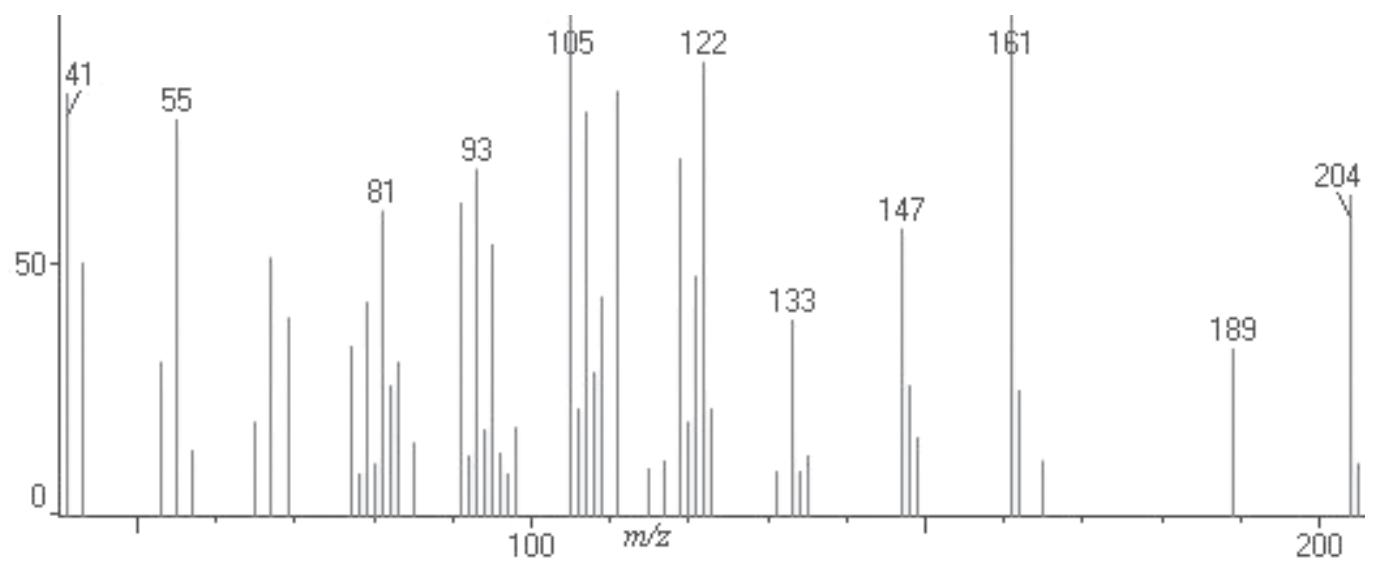

Figure S20. Mass spectrum of ledol (peak 19).

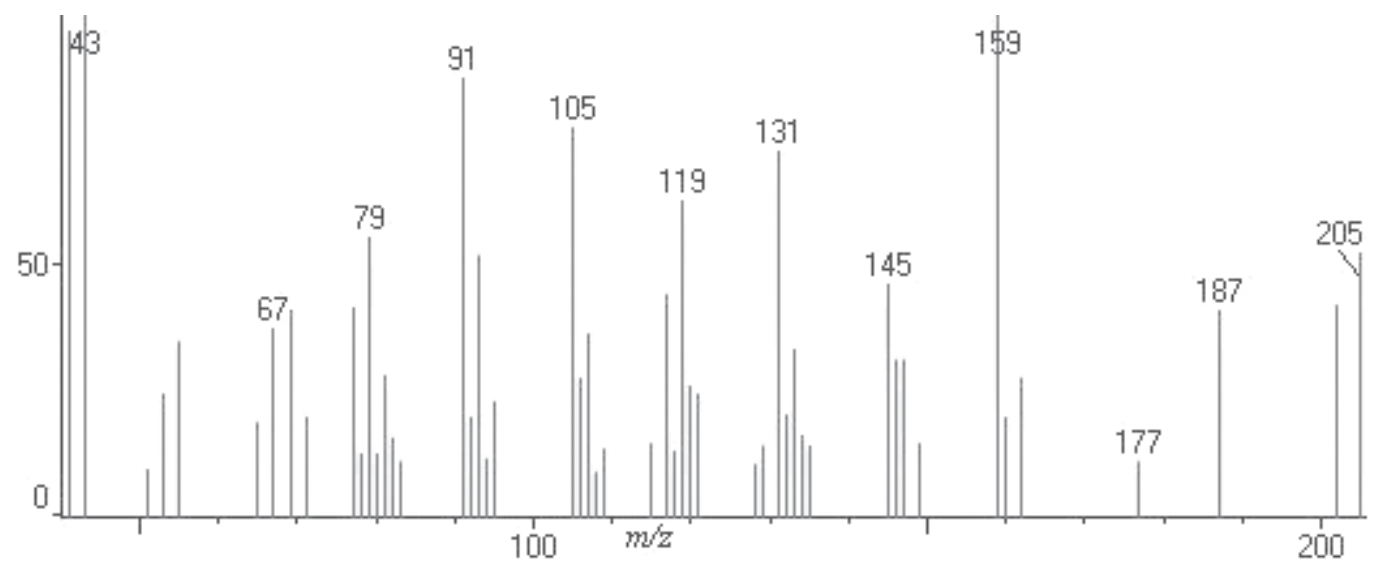

Figure S21. Mass spectrum of spathulenol (peak 20).

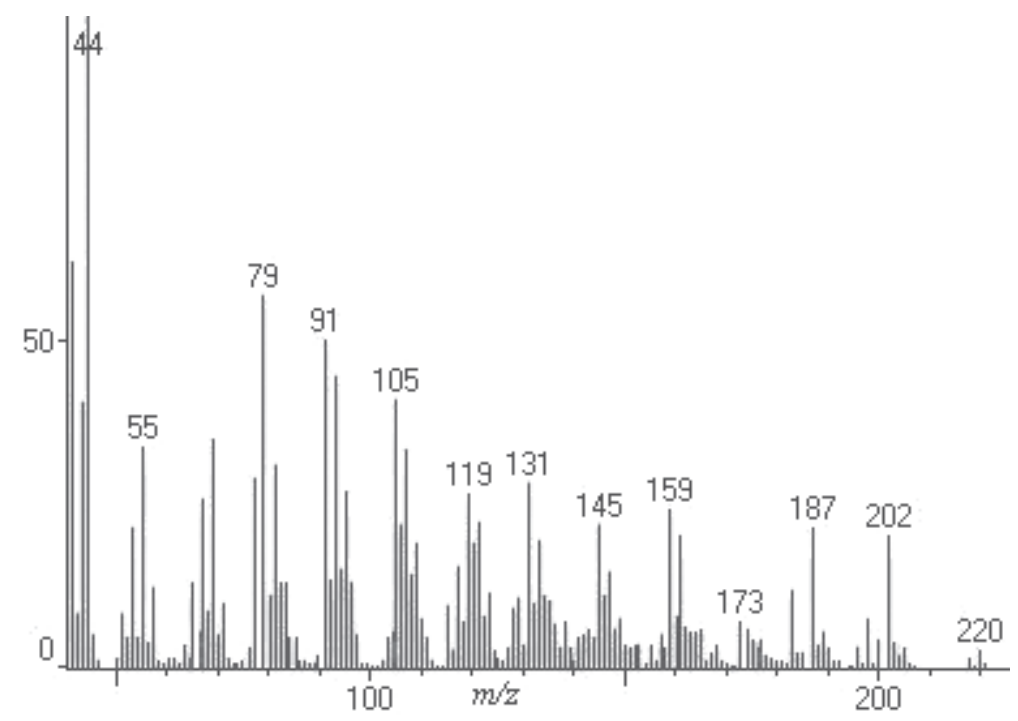

Figure S22. Mass spectrum of globulol (peak 21). 


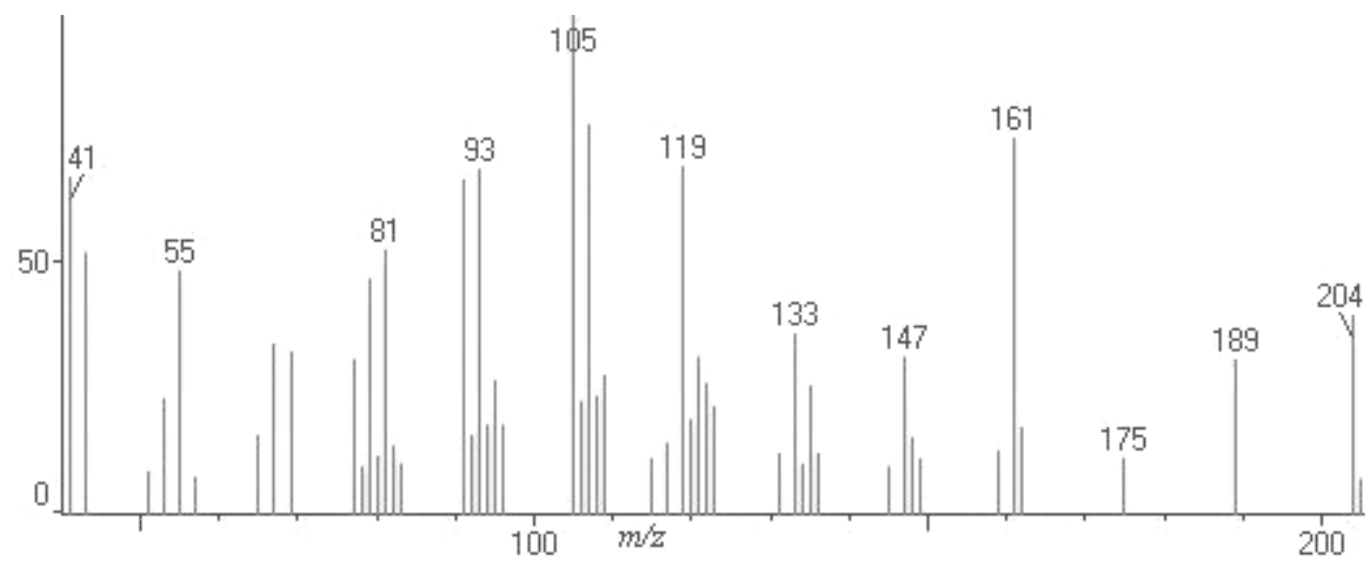

Figure S23. Mass spectrum of viridiflorol (peak 22).

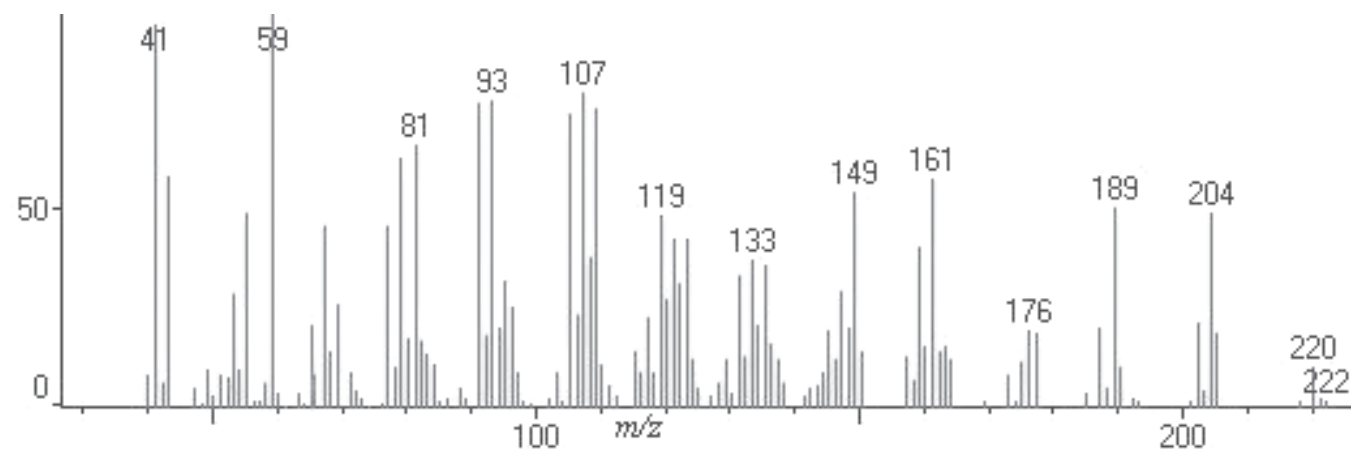

Figure S24. Mass spectrum of guaiol (peak 23).

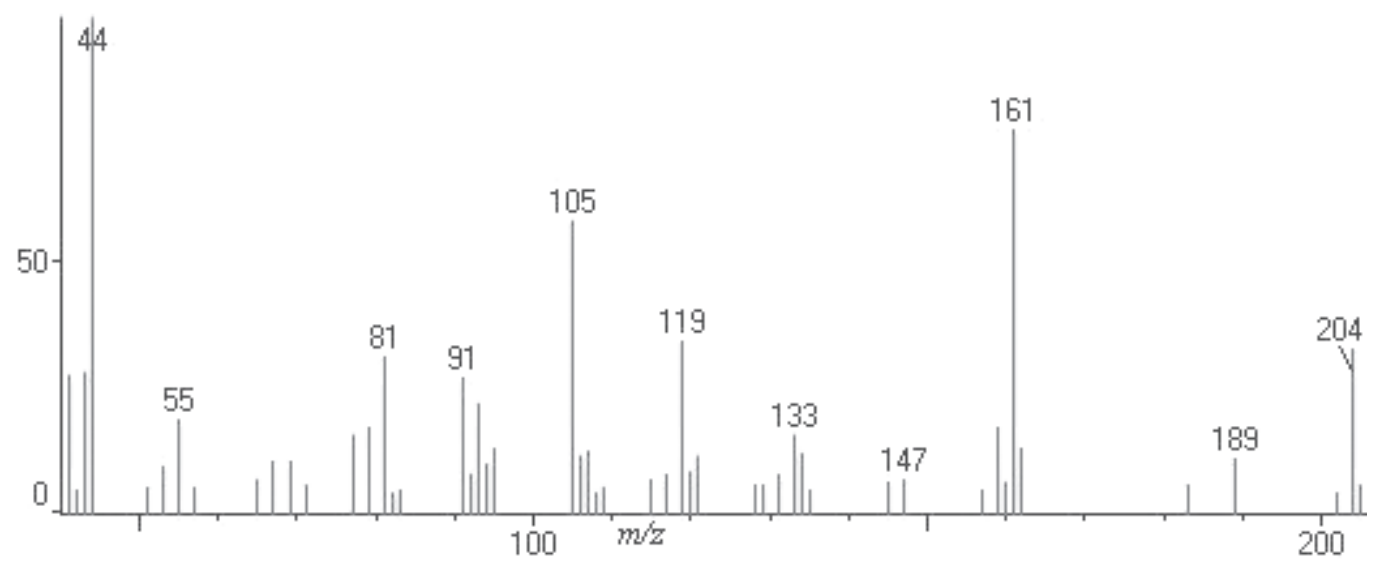

Figure S25. Mass spectrum of epi- $\alpha$-cadinol (peak 24). 


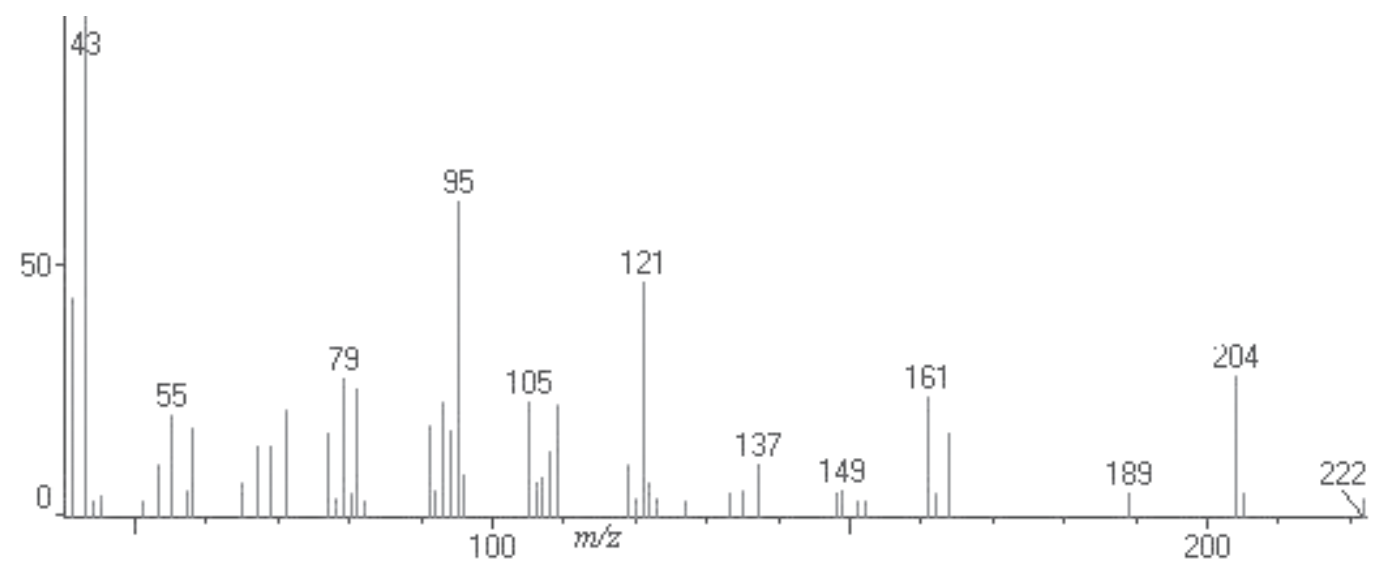

Figure S26. Mass spectrum of $\alpha$-cadinol (peak 25).

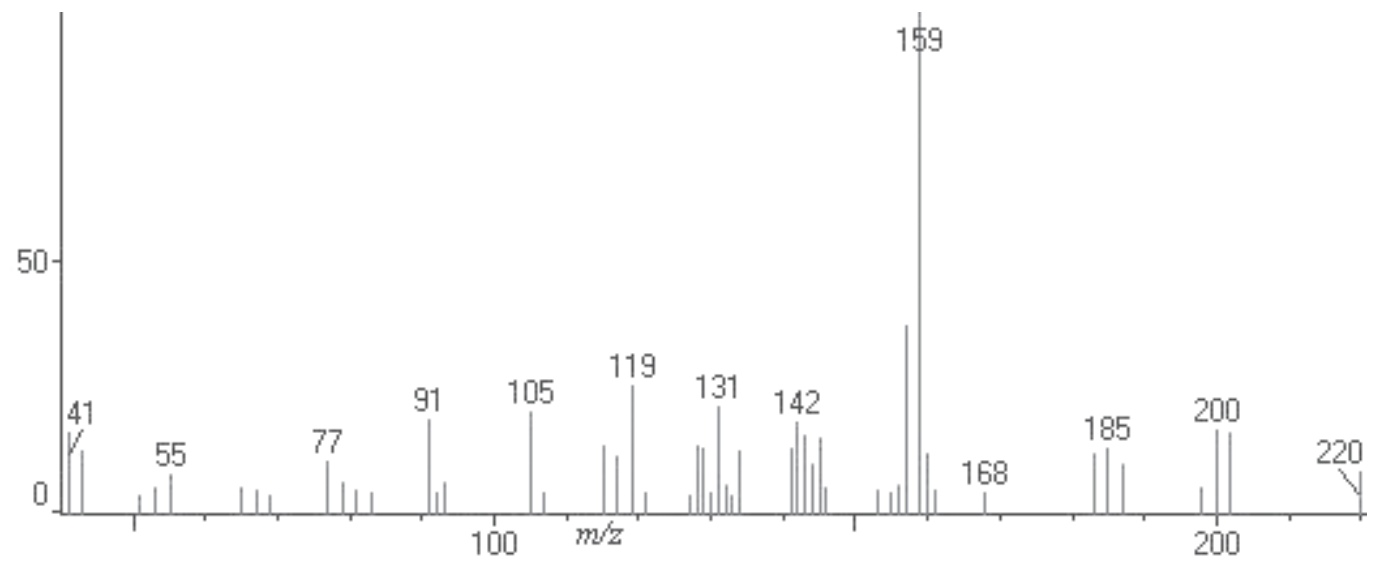

Figure S27. Mass spectrum of eudesma-4(15),7-dien-1- $\beta$-ol (peak 26). 